\title{
Shear-induced self-diffusion in non-colloidal suspensions
}

\author{
By ASIMINA SIEROU AND JOHN F. BRADY \\ Division of Chemistry and Chemical Engineering, California Institute of Technology, \\ Pasadena, CA 91125, USA
}

(Received 9 August 2001 and in revised form 17 November 2003)

Self-diffusion in a monodisperse suspension of non-Brownian particles in simple shear flow is studied using accelerated Stokesian dynamics (ASD) simulation. The availability of a much faster computational algorithm allows the study of large systems (typically of 1000 particles) and the extraction of accurate results for the complete shear-induced self-diffusivity tensor. The finite, and often large, autocorrelation time requires the mean-square displacements to be followed for very long times, which is now possible with ASD. The self-diffusivities compare favourably with the available experimental measurements when allowance is made for the finite strains sampled in the experiments. The relationship between the mean-square displacements and the diffusivities appearing in a Fokker-Planck equation when advection couples to diffusion is discussed.

\section{Introduction}

Shear-induced particle diffusion plays an important role in the behaviour of concentrated non-Brownian suspensions and is responsible for a variety of interesting rheological phenomena in such systems. The first direct experimental study of shearinduced diffusion was reported by Eckstein, Bailey \& Shapiro (1977); a variation of their experimental technique was later used by Leighton \& Acrivos (1987) and Phan \& Leighton (1999), followed by the introduction of an alternative experimental method by Breedveld et al. (1998, 2001). Most of the aforementioned experiments were limited to the determination of the self-diffusion coefficients in the velocity gradient and vorticity directions only.

Theoretical work on shear-induced diffusion is complicated by the fact that with only hydrodynamic interactions, the simplest two-body system either does not exhibit diffusive behaviour (in the velocity-gradient and vorticity directions) or exhibits a singular behaviour (an infinite diffusivity in the direction of the fluid velocity). Acrivos et al. (1992) studied the diffusion coefficient parallel to the fluid velocity by introducing a mechanism (an additional pair of particles) that results in a finite diffusivity, while Wang, Mauri \& Acrivos (1996) studied the diffusion perpendicular to the fluid velocity by examining three-particle interactions. Da Cunha \& Hinch (1996) studied the two-particle problem in the presence of surface roughness - necessary to create diffusive motion - and Brady \& Morris (1997) introduced residual Brownian motion and a hard-sphere interparticle force to break the symmetry of the two-particle limit. Marchioro \& Acrivos (2001) and Drazer et al. (2002) used Stokesian dynamics simulations to study shear-induced diffusion in concentrated suspensions and addressed the question of whether self-diffusion can be present in a purely deterministic system (e.g. in the absence of any roughness, residual Brownian motion, etc.). 
The purpose of this work is to use a recently developed algorithm for the calculation of hydrodynamic interactions among particles (Sierou \& Brady 2001) that allows the simulation of much larger systems than were previously possible. Although calculation of other rheological properties (e.g. viscosity) has proved to be accurate even with quite small systems, the calculation of the self-diffusion coefficient requires significantly larger systems. Access to systems with numbers of particles of the order of 1000 allows accurate calculation of the self-diffusion coefficient and a study of its dependence on the number of particles and the volume fraction.

The complete diffusion tensor can now be determined and simulation results for the off-diagonal components and the coefficient parallel to the fluid velocity are presented for the first time for a non-Brownian system. The presence of an external velocity field makes the determination of diffusive displacements in the flow, or longitudinal, direction difficult, and only very limited experimental results are available for these components (Breedveld 2000). Foss \& Brady (1999) calculated the longitudinal selfdiffusion coefficient for a system of Brownian particles at very high Péclet numbers by following the theoretical analysis of Morris \& Brady (1996) which suggested that the affine shearing motion could be subtracted directly at each instant in time and the diffusivity could be calculated by taking into account only the non-affine displacements. Unfortunately, as we shall show, this is not correct and the affine displacement couples with the non-affine displacement to give an additional contribution to the longitudinal long-time self-diffusivity. The calculation of the diffusion coefficient in the flow direction is still a formidable task for dilute suspensions, however, because of the strong influence of the periodicity on the particle displacements.

When advection couples to diffusion, the mean-square displacements at long time may no longer grow linearly in time. For example, in simple shear flow, the meansquare displacement in the flow direction grows as $t^{3}$, with a coefficient proportional to the diffusivity in the velocity-gradient direction (Elrick 1962). Because of this strong temporal growth, it can be very difficult to extract the underlying diffusive motion in the flow direction from measurements of the mean-square displacement. We show from an analysis of the Fokker-Planck equation both how to define the underlying shear-induced diffusivities and how to extract their values from either simulation or experiment.

In the next section, we outline the simulation method and the parameters used. In $\S 3.1$, we discuss how the diffusion coefficients are determined from both the mean-square displacements and the velocity autocorrelation function. The results for the diffusivities in the velocity-gradient and vorticity direction are presented and compared with experiment. Discrepancies between simulation and some experimental results can be attributed to the fact that most of the experimental techniques attempt to determine the self-diffusivities for very small strains where the behaviour is not yet diffusive; such a case is presented in detail in $\S 3.3$. In $\S 4$, the diffusivity in the fluid velocity direction is discussed along with the only non-zero off-diagonal component of the diffusivity tensor. Finally, we conclude in $\S 5$ with a brief summary and suggestions for future work.

\section{The simulation method}

A new implementation of the conventional Stokesian dynamics (SD) method, called accelerated Stokesian dynamics (ASD), is used in these simulations. The methodology of ASD has been laid out in Sierou \& Brady (2001) where there is a full discussion. (See also Sierou \& Brady 2002 for an application of ASD to rheology.) 
We consider the Stokes flow of a suspension of hard spheres in a fluid of viscosity $\eta$ undergoing simple shear with shear rate $\dot{\gamma}$. As has been demonstrated in the past (Melrose \& Ball 1995; Dratler \& Schowalter 1996), in the absence of Brownian motion, a repulsive interparticle force is necessary to prevent the formation of infinite clusters that 'jam' and result in excessive particle overlaps. Although the choice of this force is somewhat arbitrary, its introduction captures the behaviour of a real physical system, since the presence of residual Brownian forces or particle roughness, would also prevent the particles from touching. This force was chosen to be of the form (Bossis \& Brady 1984):

$$
\boldsymbol{F}_{p(\alpha \beta)}=F_{0} \frac{\tau \mathrm{e}^{-\tau \epsilon}}{1-\mathrm{e}^{-\tau \epsilon}} \boldsymbol{e}_{(\alpha \beta)},
$$

where $6 \pi \eta a^{2} \dot{\gamma} \boldsymbol{F}_{p(\alpha \beta)}$ is the force exerted on sphere $\alpha$ by sphere $\beta, F_{0}$ is a dimensionless constant representing the magnitude of the interparticle force, $\tau$ is related to its range, $\epsilon=r-2$ is the spacing between the surfaces of the two spheres and $\boldsymbol{e}_{(\alpha \beta)}$ is the unit vector connecting the centres of the two spheres. In the results reported here, the value of $\tau$ was set at 1000 , while $\tau F_{0}$ was chosen to be unity. Although the exact magnitude and form of this interparticle force can play an important role in the values of some rheological properties (e.g. viscosity and normal stress differences), especially for high-volume fractions, simulations using other parameters gave very similar results for the self-diffusion tensor. (At very low-volume fractions, however, where two-particle interactions dominate, the range and amplitude of the repulsive force can strongly influence the self-diffusivities (Drazer et al. 2002)).

All lengths are non-dimensionalized by the particle radius $a$, all times by the characteristic time $\dot{\gamma}^{-1}$. The time step used ranged from $\Delta t=5 \times 10^{-3}$ to $5 \times 10^{-4}$ depending on the volume fraction (higher-volume fractions required smaller time steps). A fourthorder Adams-Bashforth integration scheme was used to update the particle positions.

\section{The self-diffusivities in the velocity gradient $\left(D_{y y}\right)$ and vorticity $\left(D_{z z}\right)$ directions}

\subsection{Numerical determination of the self-diffusivities}

We now describe the calculation of the self-diffusion coefficients in the velocitygradient and vorticity directions. (The calculation of the diffusivity in the fluid velocity direction is subject to further limitations and will be described in the following section.) Dimensional arguments show that the self-diffusivities scale as $\dot{\gamma} a^{2}$, as these are the only time and length scales in the system (in the absence of interparticle forces). All diffusivities reported are therefore normalized by $\dot{\gamma} a^{2}$.

Let $x, y$ and $z$ denote the directions of the flow, the velocity-gradient and the vorticity, respectively. Then, for a suspension undergoing simple shear flow in the $x$-direction with velocity gradient in the $y$-direction, the mean-square displacements will grow with time according to

$$
\begin{aligned}
\langle y(t) y(t)\rangle & \sim 2 D_{y y} t, \\
\langle z(t) z(t)\rangle & \sim 2 D_{z z} t,
\end{aligned}
$$

at long times after diffusive motion has been established. Here, and hereinafter, the angle brackets denote an average over all particles in the system, while $D_{y y}$ and $D_{z z}$ denote the self-diffusivities in the velocity-gradient and vorticity directions, respectively. The self-diffusivities are defined as the time rate of change of half the mean-square displacements, and can be calculated in a straightforward manner by a number of different, but equivalent, methods. 

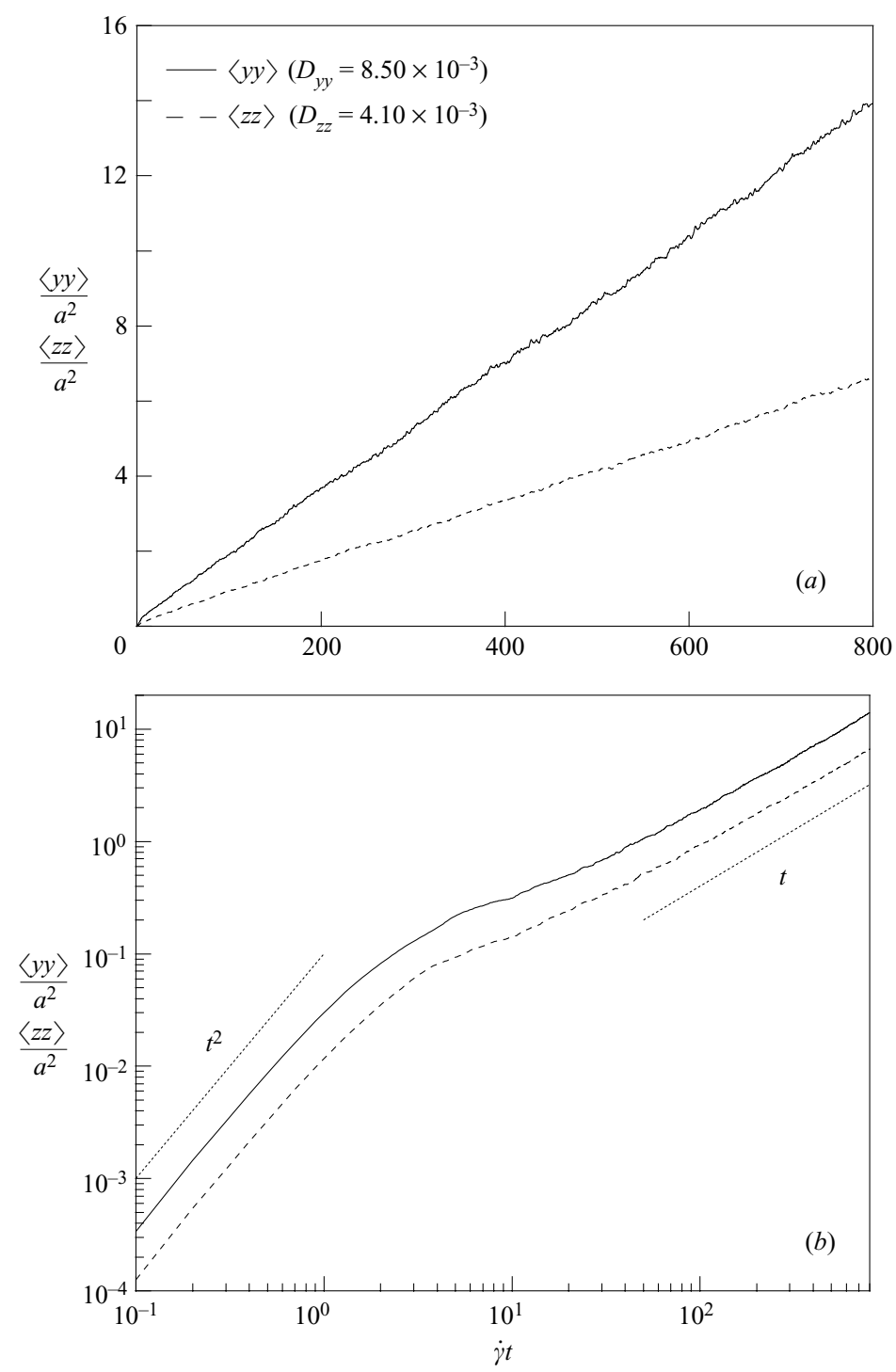

FIgURE 1. The mean-square displacement curves $\langle y y\rangle$ and $\langle z z\rangle$ as a function of time (or strain) $\dot{\gamma} t$ for a system of $N=1000, \phi=0.20$ for strains up to 800 , in $(a)$ a linear and $(b)$ a $\log -\log$ plot. The values of the resulting diffusivities are also given. In this and subsequent figures, all mean-square displacements are in units of $a^{2}$, and all diffusivities are in units of $\dot{\gamma} a^{2}$.

First, the mean-square displacement curve can be constructed for a long simulation run. Using a large number of particles, this approach results in a mean-square displacement curve that is linear in time at long times, as shown in figure 1 for a system with $N=1000$ at volume fraction $\phi=0.2$. It is clear from the logarithmic plot that, at very short times, the mean-square displacement shows a quadratic temporal behaviour, while after a strain of about 20 the final linear regime sets in and continues until the end of the run. The quadratic behaviour corresponds to the regime where the particle motion is deterministic and still strongly correlated - the diffusive behaviour has not yet been established. It should be noted that, although after a strain of about 

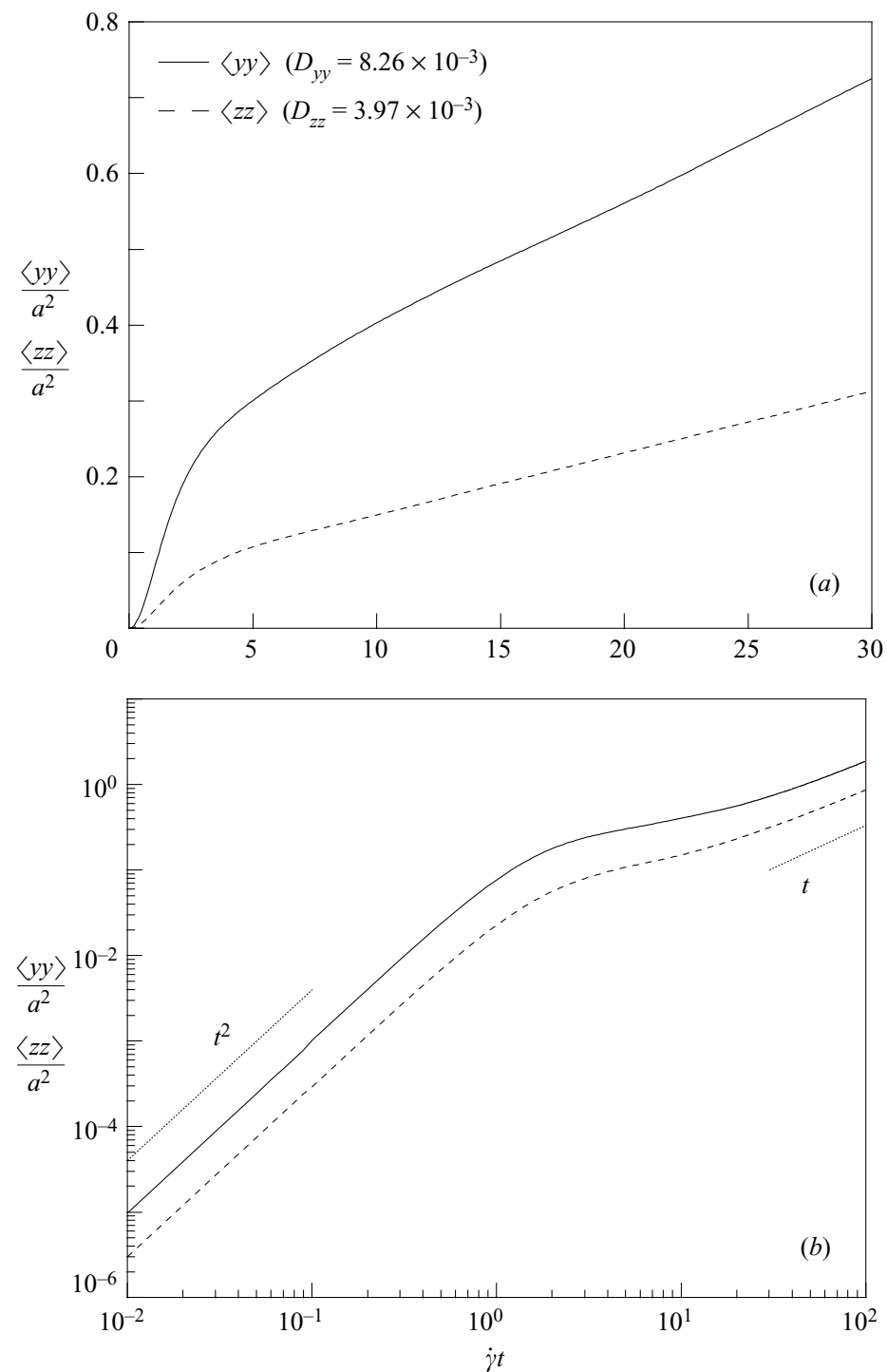

FiguRE 2. The mean-square displacement curves $\langle y y\rangle$ and $\langle z z\rangle$ as a function of time (or strain) $\dot{\gamma} t$ for a system of $N=1000, \phi=0.20$ averaged over a total strain of $(a) 30$ and $(b) 100$. The values of the resulting diffusivities are also given.

one the quadratic scaling no longer holds, a transitional region exists up to strains of at least 10 before a clear linear behaviour is evident. The slope of the resulting curve for $t>20$ can be readily evaluated and the resulting diffusivity is given in figure 1 .

Second, this same long simulation run can be split into shorter independent runs, and the resulting mean-square displacement curves from each shorter run averaged. Although an infinite time limit is implied for obtaining the long-time self-diffusivity, the particle does not necessarily need to travel a very long distance to reach this longtime asymptote; it simply needs to encounter enough particle-particle interactions to sample the dynamic microstructure and 'forget' its initial configuration and velocity. Figure 2 is for the same simulations as in figure 1, but now over a shorter time interval. 
The long run of the previous case was split into smaller runs of 30-100 strains, the results averaged and presented again in linear and logarithmic plots. Because of the increased statistical data, the slope of the mean-square displacement curve can be evaluated with greater accuracy than before. The final result for the diffusivity is very close to the value reported in figure 1 and within the statistical accuracy of the data. Although the linear behaviour of the curves in the short and long runs is identical, the initial transient behaviour, as is apparent from the logarithmic plots, can be quantitatively different (qualitatively, a $t^{2}$ behaviour is apparent for both cases). This is because for the shorter runs the sequence of independent configurations are all sampled after the system has reached its steady-state configuration, while the beginning of the overall mean-square displacement curve in figure 1 corresponds to an initially random configuration. As a result, a different quadratic behaviour is seen.

Finally, the diffusivities can be evaluated from the integral of the velocity autocorrelation function using the well-known expression:

$$
D_{y y}=\int_{0}^{\infty} \lim _{t \rightarrow \infty}\left\langle u_{y}(\tau+t) u_{y}(t)\right\rangle \mathrm{d} \tau,
$$

where $u_{y}$ is replaced by $u_{z}$ for $D_{z z}$. The time it takes for the velocity autocorrelation to decay to zero corresponds to the time it takes the mean-square displacement curve to reach the linear regime; for the diffusivities to be evaluated correctly, the integral must be computed for at least that minimum time. As shown by Marchioro \& Acrivos (2001), the velocity autocorrelation has a negative contribution that must be included to obtain the correct diffusivity. For the case shown in figure 1, the integral of the velocity autocorrelation function gave $D_{y y}=8.30 \times 10^{-3}$ and $D_{z z}=3.96 \times 10^{-3}$.

We could also improve the statistics by averaging over a number of realizations or initial conditions; however, with the large number of particles used and the long simulation times, our results appear to be sufficiently accurate.

The three approaches to determine the self-diffusivity gave consistent results for all volume fractions studied. The time necessary for the diffusive behaviour to be reached is a strong function of the volume fraction, and, as has been reported in the past, increases with decreasing volume fraction. For very dilute suspensions, the particle interactions are limited and a larger strain is necessary until the particles have sampled a sufficiently large number of independent collisions and the linear regime is reached.

Before we proceed to the presentation of the majority of our simulation results, it is worth mentioning briefly how we define their statistical properties. The values of the diffusivities obtained from the three aforementioned approaches are in all cases in excellent agreement, so their simple average is used as the reported value of the diffusivity. The calculation of the standard deviation, however, needs to be discussed in more detail. The error associated with the calculation of any element of the self-diffusion tensor can result from one (or more) of the following sources. First, errors derived from the solution of the equations of motion - the assumptions used in the development of ASD combined with the 'standard' numerical errors present in any numerical calculation (finite time steps, machine accuracy, etc.) - always lead to a numerical error in the calculation of any property. The statistical nature of the particle displacements and the fact that the magnitude of the numerical error is usually small makes the numerical inaccuracies of very little importance and therefore we shall not consider them further. Secondly, there is the uncertainty associated with the number of independent mean-square displacement curves available. The meansquare displacement curves shown in figures 1 and 2 are already averaged over 
all particles, and, for the case of figure 2, all the shorter independent runs are also averaged. In order to obtain the correct values for the self-diffusion tensor, it must be assured that a sufficient number of mean-square displacement curves have been sampled, or in other words, that all possible particle interactions have been sampled. This can be assured by examining systems with large $N$, or a large number of configurations of systems with smaller $N$, as has been suggested in the past (Foss \& Brady 1999). The calculation of the error then corresponds to grouping particles/configurations together and calculating the standard deviation of the resulting measurements. This is the error routinely reported in our results and is calculated either as a temporal average over smaller segments of the mean-square displacement curve for the long runs of figure 1, or as an average over different groups of configurations for the case of figure 2. (Note that figure 2 presents the average mean-square displacement curve over all possible configurations and particles for a given run; only the mean can be calculated from this curve.) Finally, the calculation of the slope of the mean-square displacement curve, or the numerical integration of the velocity autocorrelation function, introduces a numerical error. In addition to any uncertainty associated with the numerical calculation, the most important issue is the correct time regime (or strain) over which to determine the diffusivities. Very long tails in the velocity autocorrelation function can be erroneously discarded and visual observation of when the process becomes linear can be misleading and result in large errors. Although we believe that this source of error can be potentially very important, we have tried to make a conservative estimate in our calculation of the minimum strain needed for diffusive behaviour to set in, and are confident that the error from this source is not significant. Therefore, for the remainder of this paper the error bars reported correspond to uncertainties associated with simply the volume of the statistical data, uncertainties that for most cases are less that $10 \%$. Finally, the last source of uncertainty, system size and the effect of the periodic unit cell, will be discussed in more detail below.

\subsection{The dependence on the number of particles $N$}

The introduction of a new faster methodology allows the calculation of the selfdiffusion coefficients for numbers of particles of the order of $10^{3}$, and we can therefore present a reliable analysis of the dependence of the diffusivities on the number of particles, $N$. Figure 3 shows the dependence of $D_{y y}$ on $N$, for the case of $\phi=0.35$ (the interparticle force, defined in (2.1) with the parameters mentioned, is always present unless otherwise noted). There is a rather sharp increase in the diffusivity for small values of $N(N<100)$, followed by a levelling off. The same trend is apparent for all elements of the self-diffusion tensor. No theoretical expression is available for the $N$ dependence of the long-time self-diffusivity, and so in figure 3 we simply present a $N^{-1}$ best fit that seems to describe the data satisfactorily. Plotting the individual mean-square displacements (not shown) for different numbers of particles also verifies that for $N>100$ all curves are statistically indistinguishable. A possible explanation of this behaviour may lie in the fact that after the size of the cell is sufficiently large (or $N$ is sufficiently large), all of the important particle interactions are included and therefore the number of particles plays no role (other than increasing the statistical accuracy, i.e. giving curves that appear smoother). When the number of particles is very small, the corresponding size of the cubic unit cell is also small (for $N=27$, $L=6.86 a$ for $\phi=0.35$ ). This means that particle interactions for separations greater than $L$ occur through periodic images, which may explain the smaller diffusivities observed for very small number of particles. (As discussed in $\S 4.2$ a similar, but much 


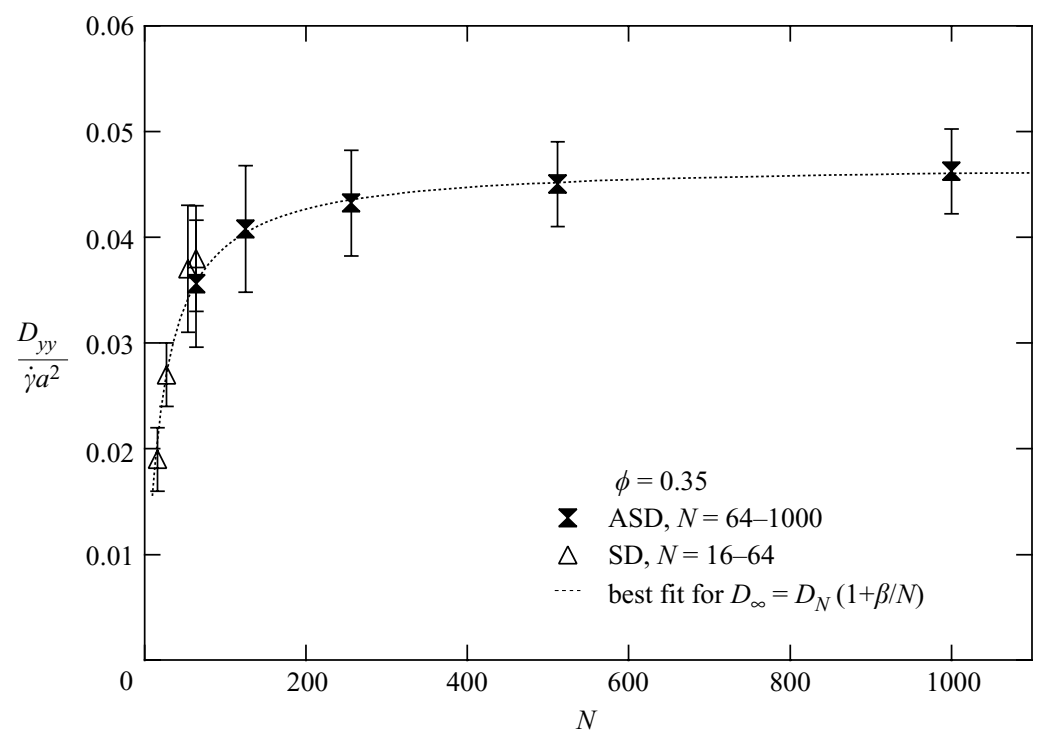

FIGURE 3. The dependence of $D_{y y}$ on the number of particles $N$, with $N$ ranging from 16 to 1000 , for $\phi=0.35$. A very sharp increase is observed for small volume fractions, followed by a relatively constant value.

$\begin{array}{ccc}\phi & D_{y y} & D_{z z} \\ 0.10 & 0.0017 \pm 0.0003 & 0.0011 \pm 0.0002 \\ 0.15 & 0.0045 \pm 0.0006 & 0.0024 \pm 0.0004 \\ 0.20 & 0.0084 \pm 0.0010 & 0.0040 \pm 0.0006 \\ 0.25 & 0.0171 \pm 0.0020 & 0.0070 \pm 0.0007 \\ 0.30 & 0.0310 \pm 0.0040 & 0.0117 \pm 0.0010 \\ 0.35 & 0.0460 \pm 0.0050 & 0.0185 \pm 0.0020 \\ 0.40 & 0.0620 \pm 0.0060 & 0.0290 \pm 0.0030 \\ 0.45 & 0.0583 \pm 0.0070 & 0.0450 \pm 0.0040 \\ 0.50 & 0.0580 \pm 0.0070 & 0.0520 \pm 0.0050\end{array}$

TABLE 1 . The dependence of $D_{y y}$ and $D_{z z}$ on the volume fraction $\phi$ for $N=1000$.

more apparent, effect of the size of the unit cell is present in the calculation of $D_{x x}$ for low volume fractions.) For the remainder of this paper all results will correspond to systems of $N=1000$ particles and no further corrections to the infinite system limit will be attempted since, as is apparent in figure 3, such corrections would be very small and within the statistical accuracy of the calculations.

\subsection{The dependence on the volume fraction}

Figures 4 and 5 and table 1 show the dependence of $D_{y y}$ and $D_{z z}$ on the volume fraction $\phi$. Although qualitatively most sets of experimental or simulation results are in agreement, quantitatively discrepancies are present that need to be explained and examined in more detail. As was mentioned in $\S 3.1$, the notion that the long-time self-diffusivities can be calculated for strains that are not particularly long has been used, and most of the recent experimental and simulation results presented in figures 4 and 5 are calculated over a large number of configurations, but for relatively small strains (Breedveld et al. 1998, 2001; Foss \& Brady 1999). Unfortunately, as shown 


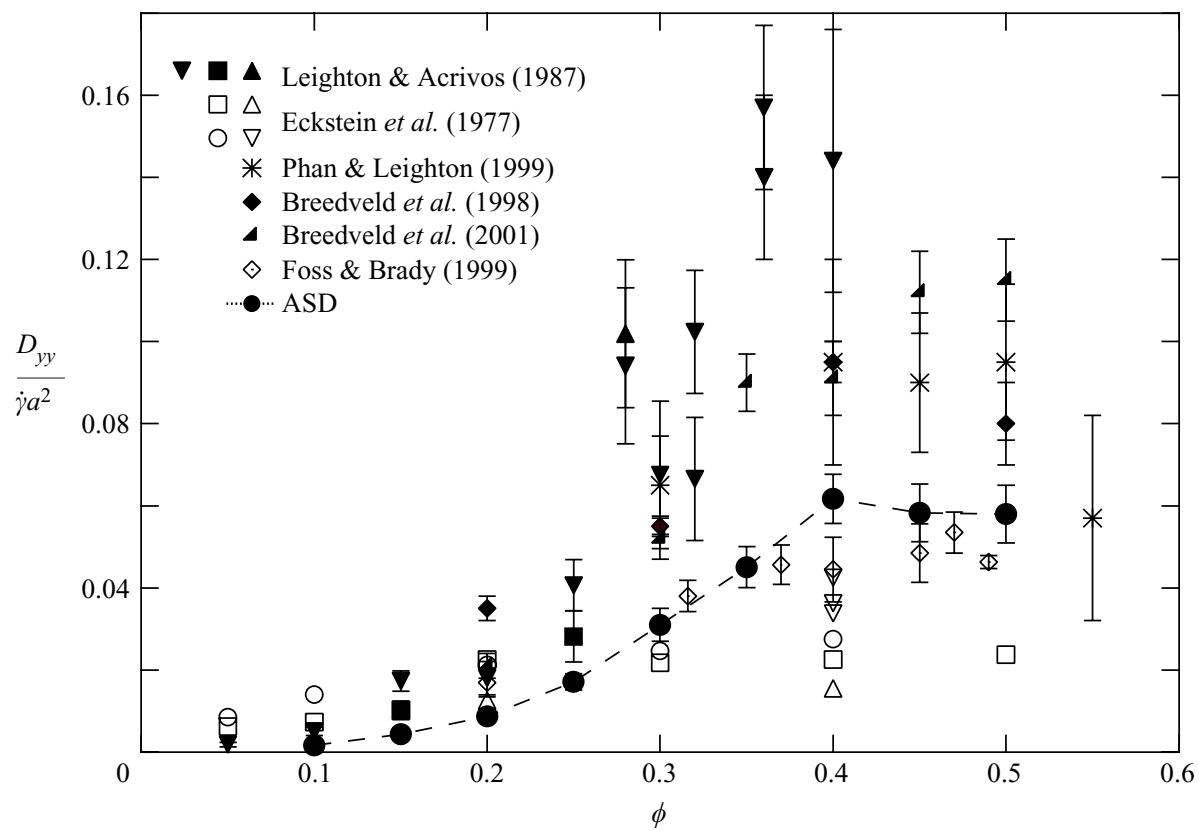

FIGURE 4. The dependence of $D_{y y}$ on the volume fraction $\phi$. Accelerated Stokesian dynamics (ASD) results are for a system of $N=1000$ and compared with a number of experimental and simulation results.

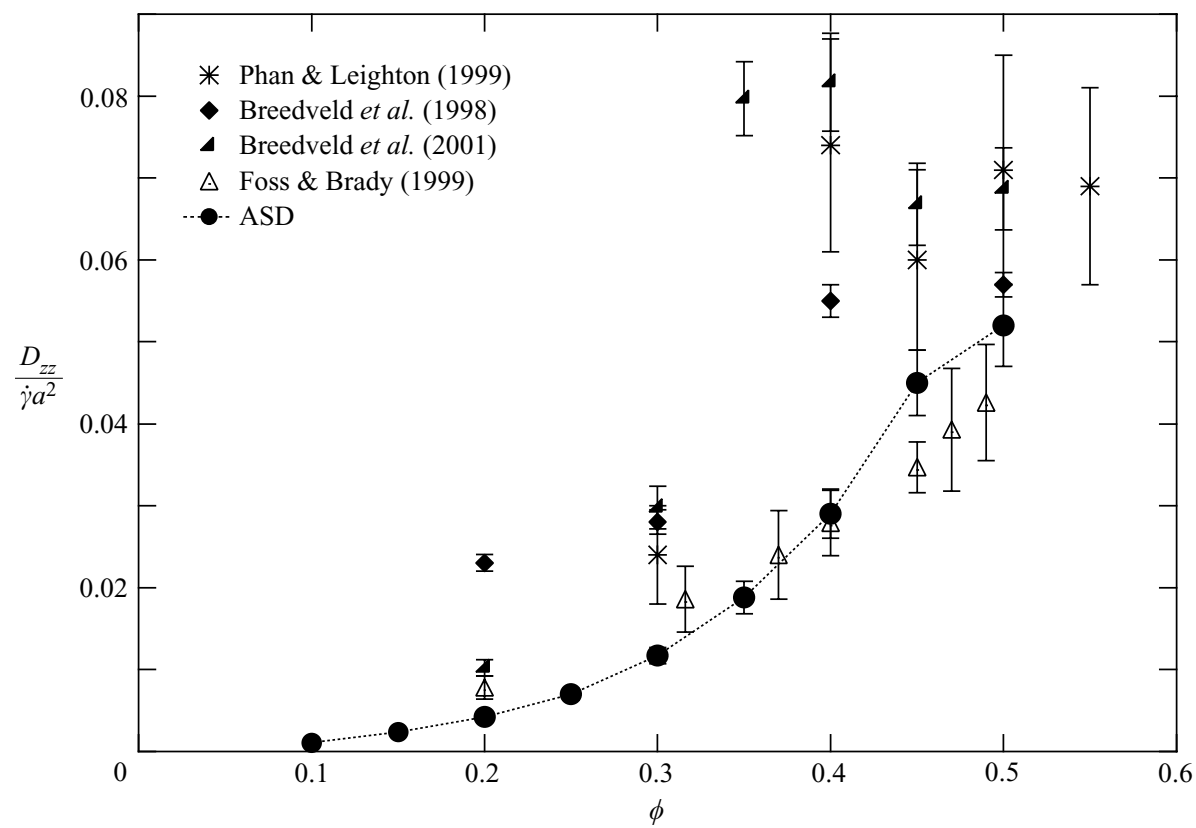

FIGURE 5. The dependence of $D_{z z}$ on the volume fraction $\phi$. Accelerated Stokesian dynamics (ASD) results are for a system of $N=1000$ and compared with a number of experimental and simulation results. 

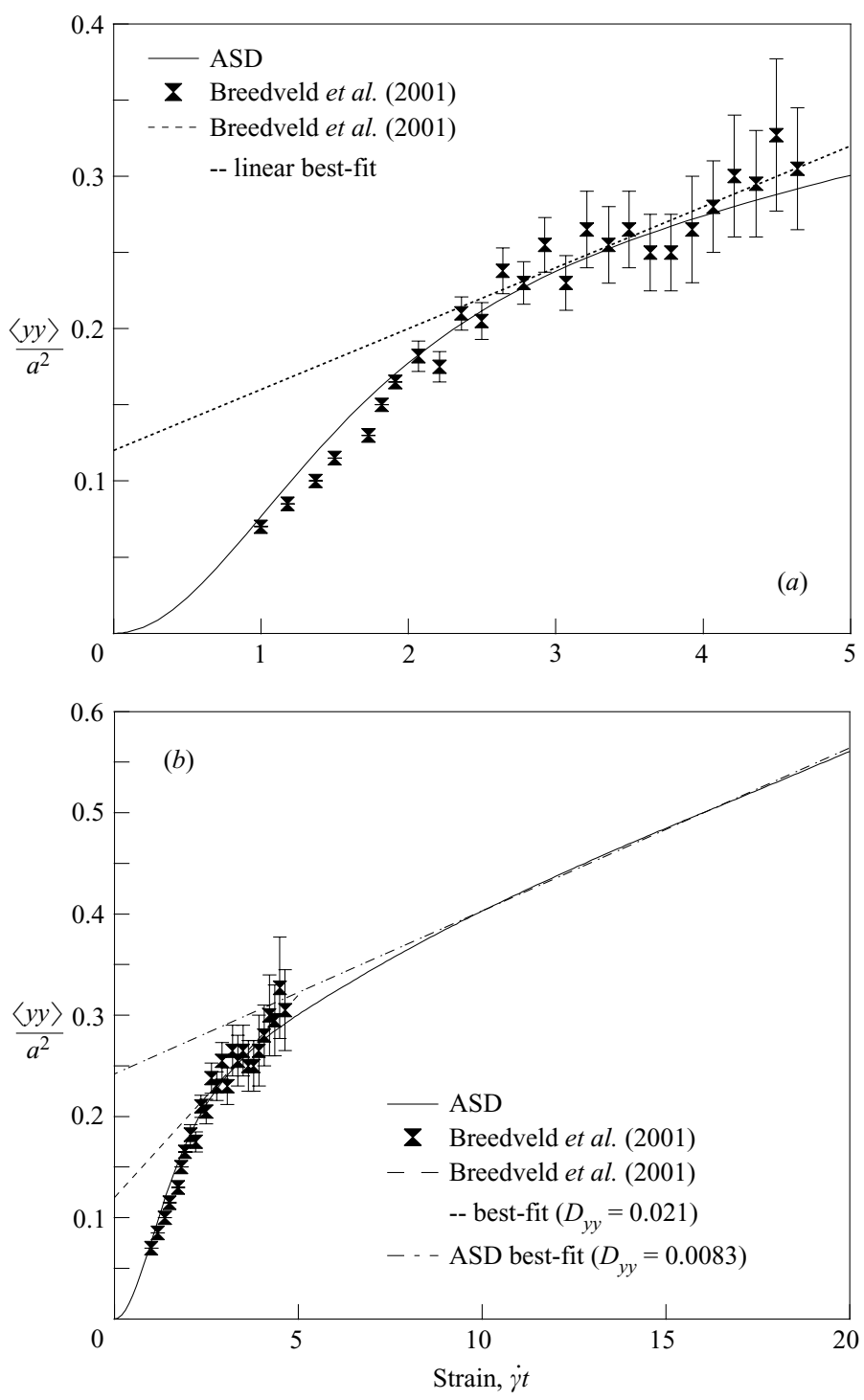

FIGURE 6. The mean-square displacement $\langle y y\rangle$ from ASD simulations for $\phi=0.20, N=1000$ is compared with the equivalent results given by Breedveld et al. (2001) for strains of $(a) 5$ and $(b)$ 20. The resulting diffusivities and slopes of the mean-square displacement curve are also shown.

below and as was discussed by Marchioro \& Acrivos (2001), the strains used for these studies are too small and therefore do not correspond to the linear regime, as a result they overestimate the diffusivity.

Figure 6(a) is adapted from Breedveld et al. (2001) and shows the mean-square displacement curve, $\langle y y\rangle$, for $\phi=0.20$ for strains up to 5. (We choose to examine the case of a relatively small volume fraction in detail because, as is apparent in figure (8) of Breedveld et al. (2001), the accuracy in their determination of the meansquare displacement curve appears to be greatly reduced when the volume fraction is increased owing to limitations of the experimental technique; this is not observed 


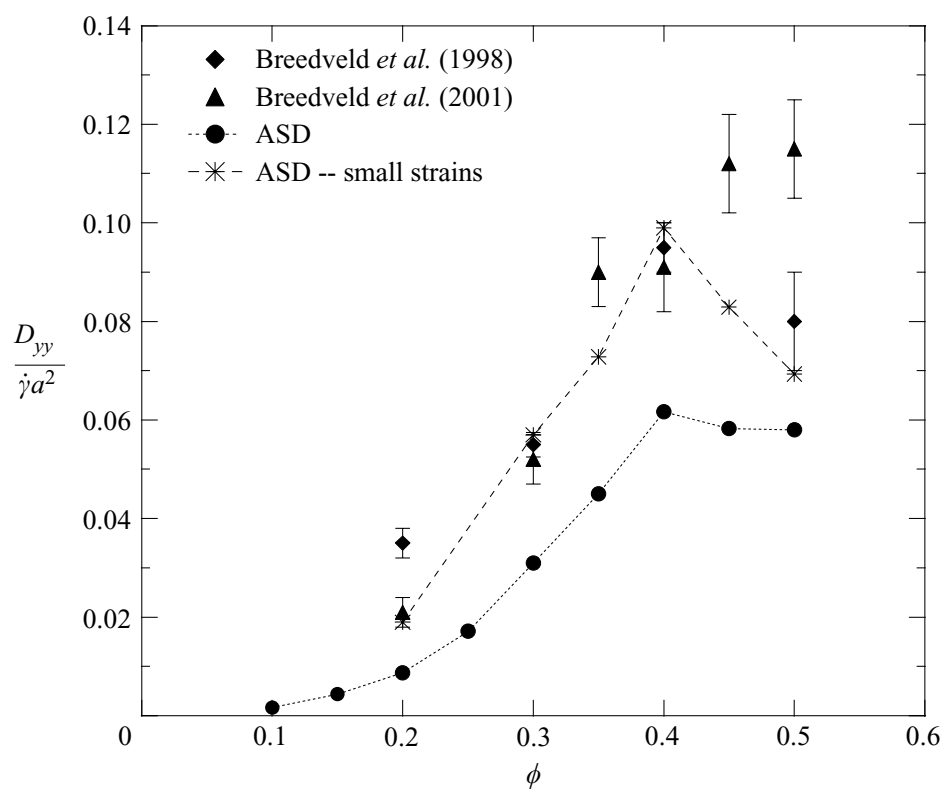

FIGURE 7. The self-diffusion coefficient $D_{y y}$ as calculated from ASD for long and short strains and compared with the experimental results (corresponding to the same short strains) of Breedveld et al. (2001).

in the numerical simulations.) The dashed curve represents the 'linear-fit' performed by Breedveld et al. (2001); the value of the diffusivity that they obtain is also given. The solid line corresponds to our mean-square displacement for the same volume fraction for a system of $N=1000$ particles. The agreement between the two curves is remarkably good over the entire range of experimentally accessible strains. Although this is the case for the mean-square displacement curves, the resulting diffusivities are not in good agreement and the value reported by Breedveld et al. (2001) is over twice as large as the value we calculate. The reason for this is apparent in figure $6(b)$, where the same mean-square displacement curve is presented but now for strains up to 20 . It is now clear that the linear regime has not yet been reached at a strain of 5, and strains of at least 10 need to be sampled for the long-time diffusive behaviour to be reached. In figure 6(b), we also show the change in slope in the $\langle y y\rangle$ curve and how, if the strain is not sufficiently long, the slope simply corresponds to a transitional regime between the $t^{2}$ and $t$ behaviours and overestimates the diffusivity. The same conclusion is reached by studying the velocity autocorrelation function: the long tail present in the velocity autocorrelation function is not taken into account when short strains are used and, since the velocity autocorrelation function is negative at those strains, the value of the diffusivity is overestimated (Marchioro \& Acrivos 2001).

Figure 7 presents our simulation results, but now instead of calculating the slope of the displacement curves in the linear regime, we calculate them for the same strains reported by V. Breedveld (personal communication). We now observe a much better agreement between the numerical and experimental data for all volume fractions (although for larger volume fractions, some discrepancies are still present).

Although the limitation on strain in the experiments of Breedveld et al. (2001) explains the difference between simulation and these experiments, this is not necessarily the case for the other experimental results. Data by Leighton \& Acrivos 


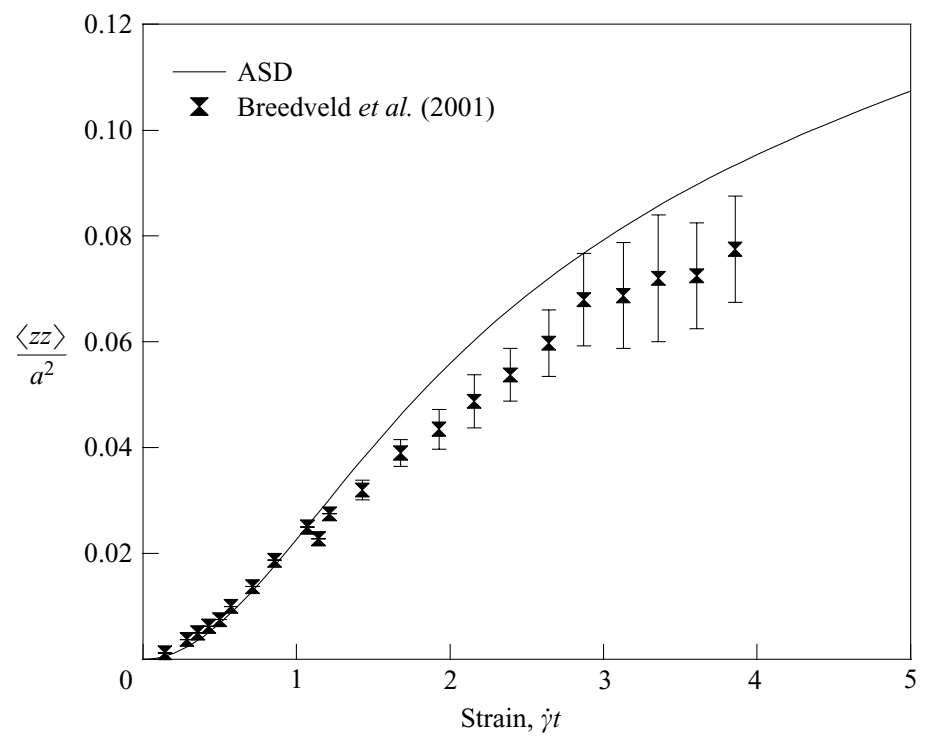

FIGURE 8. The mean-square displacement $\langle z z\rangle$ as calculated from ASD simulations for $\phi=0.20, N=1000$ is compared with the equivalent results given by Breedveld et al. (2001) for strains of 5 .

(1987) and Phan \& Leighton (1999) are calculated for larger strains, but with a different method that collects data on relatively few particles/realizations, and the origin of the differences between these experiments and the simulations is not known.

We also wish to briefly comment here on the data by Foss \& Brady (1999). In their approach, very small strains were also used, strains that according to figure $6(b)$ should overestimate the resulting mean-square displacement coefficient significantly. As is apparent from figure 4, however, this is not the case and our results are in general agreement with the results reported by Foss \& Brady (1999). We believe that this is the result of the very small systems used by Foss \& Brady (1999); $N=27$ for all of their simulations. As was mentioned in the previous subsection, a very strong $N$ dependence is present for such a small number of particles and the diffusivities are underestimated significantly. It is the combination of these two sources of error the very small number of particles and the very small strains used - that cancel each other and lead to a diffusivity of the correct magnitude.

Figure 8 presents the $z z$ mean-square displacement curve for $\phi=0.20$ as given by Breedveld et al. (2001) compared with our simulation results; again the agreement is reasonable, although not nearly as good as in figure 7. As shown in figure 9, the agreement between the short strain simulations and the experimental data is not as good for higher volume fractions as was the case for $D_{y y}$. This seems to be a consequence of the different functional dependence on the volume fraction (see below for more detail); in our data, $D_{z z}$ is an increasing function of the volume fraction (and as a result so are the values calculated for smaller strains), while Breedveld's data show a very sharp increase followed by a plateau. We have no explanation for this qualitative difference. It seems that although the method developed by Breedveld et al. $(1998,2001)$ has some very attractive characteristics that can reproduce accurate results for small strains, larger strains are needed to capture the long-time diffusive behaviour and some adjustments are necessary in the method to allow for larger observation windows. 


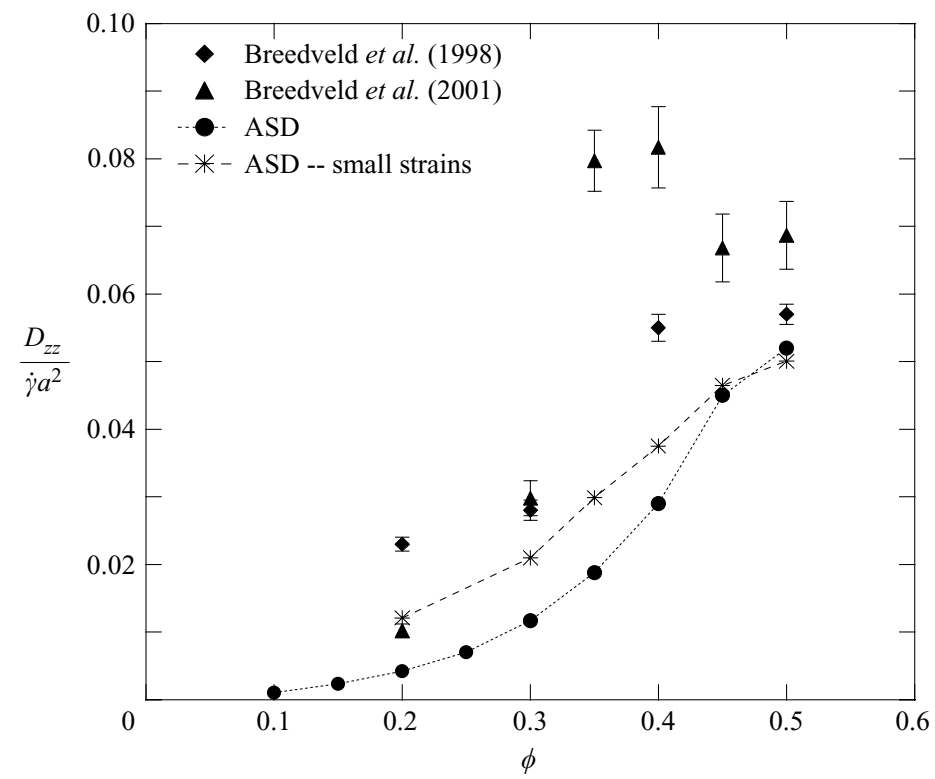

FIGURE 9. The self-diffusion coefficient $D_{z z}$ as calculated from ASD for long strains, short strains and compared with the experimental results (corresponding to the same short strains) given by Breedveld et al. (2001).

We now offer a brief discussion of the qualitative behaviour of $D_{y y}$ and $D_{z z}$ as a function of volume fraction. For small volume fractions, $\phi<0.30$, a $\phi^{2}$ behaviour is evident for both diffusivities. This is expected since, in the absence of any interparticle force, the interactions between only two particles lead to zero net displacements (the particles always return to their original streamlines), and the presence of a third particle is necessary to generate non-zero displacements leading to a diffusivity of $O\left(\phi^{2}\right)$. As shown by da Cunha \& Hinch (1996), however, with an interparticle force, a net displacement in the velocity-gradient and vorticity directions can be achieved with only two particles, leading to an $O(\phi)$ dependence of the diffusivity. For the range of the interparticle force used here and the minimum separations that result, the size of this $O(\phi)$ correction is extremely small and is overwhelmed by the displacements caused by the presence of a third particle. Evidently, the three-particle effects are significant even at the lowest volume fraction studied $(\phi=0.10)$. Much lower-volume fractions would be necessary to capture and verify the order $\phi$ correction to the diffusivity. We briefly note here that it is not clear whether the presence of an interparticle force is necessary for diffusive motion to appear. It has been suggested in the past (Marchioro \& Acrivos 2001) that even in the absence of an interparticle force, diffusive-like characteristics could be present owing to the chaotic nature of the many-body hydrodynamic interactions. If that is indeed the case, the order $\phi$ correction to the diffusivity might not be dominant even for extremely small volume fractions (Drazer et al. 2002).

As is apparent from figure 4, and in qualitative agreement with most experimental results, the diffusivity in the velocity-gradient direction appears to plateau at volume fractions about $\phi=0.35-0.40$. This is not the case for the diffusivity in the vorticity direction however, which is seen to increase roughly as $\phi^{2}$ for all volume fractions studied. The possible exception being $\phi=0.50$, where the increase is less apparent; 
the experimental results for this case suggest the presence of an earlier plateau in the value of $D_{z z}$. The presence of such a plateau in either $D_{y y}$ or $D_{z z}$ has not been explained.

\section{The longitudinal $\left(D_{x x}\right)$ and off-diagonal $\left(D_{x y}\right)$ self-diffusivities}

\subsection{Theoretical approach}

The relationship between the complete diffusivity tensor (including $D_{x x}$ and $D_{x y}$ $\left[=D_{y x}\right]$ ) and the mean-square displacement for a Brownian particle suspended in a fluid undergoing simple shear flow has been the subject of numerous studies. Elrick (1962) calculated the $\langle x x\rangle$ and $\langle x y\rangle$ mean-square displacements for such a Brownian particle and found them to grow in time according to:

$$
\begin{aligned}
& \langle x x\rangle=2 D_{x x} t+2 D_{x y} \dot{\gamma} t^{2}+\frac{2}{3} D_{y y} \dot{\gamma}^{2} t^{3}, \\
& \langle x y\rangle=2 D_{x y} t+D_{y y} \dot{\gamma} t^{2} .
\end{aligned}
$$

Diffusive motion in the $y$-direction, $D_{y y}$, couples to the advective flow in the $x$ direction to give a mean-square displacement in the flow direction that grows cubically in time. In obtaining (4.1), it is assumed that all diffusivities are constant in time and that the 'random' Brownian step the particle takes is diffusive at all times.

Using (4.1) for a system of non-Brownian particles poses further limitations, e.g. the motion in the $y$-direction is not diffusive at short times and therefore (4.1) can only be valid at very long times where the $t^{3}$ (or $t^{2}$ ) behaviour will dominate and the linear term $D_{x x} t$ (or $D_{x y} t$ ) will be difficult to determine accurately. It is therefore desirable to derive an alternative formulation that not only allows the accurate calculation of the diffusivities, but also serves as a proof of the validity of (4.1) for a system of non-Brownian particles. Following the equations of motion for our system, we show how to calculate the corresponding mean-square displacements and correctly deduce the diffusion coefficients. by:

The position of a given particle (initially at the origin) in the $x$-direction is given

$$
\begin{aligned}
x_{\alpha}(t) & =\int_{0}^{t} U_{x, \alpha}(\tau) \mathrm{d} \tau=\int_{0}^{t}\left(U_{x, \alpha}^{h}(\tau)+U_{x, \alpha}^{\infty}(\tau)\right) \mathrm{d} \tau \\
& =X_{\alpha}^{h}+\int_{0}^{t} \dot{\gamma} y_{\alpha}(\tau) \mathrm{d} \tau,
\end{aligned}
$$

where, $U_{x, \alpha}^{h}$ and $U_{x, \alpha}^{\infty}$ correspond to the instantaneous velocities of particle $\alpha$ owing to the presence of all the other particles and to the external velocity field, respectively. For the case of simple shear flow, the external velocity field at the centre of the particle is simply $U_{x, \alpha}^{\infty}=\dot{\gamma} y_{\alpha}$. In a simulation where the position of each particle is known exactly and the particle velocities are already decomposed into an affine $\left(\boldsymbol{U}^{\infty}\right)$ and a non-affine $\left(\boldsymbol{U}^{h}\right)$ part, each term of equation (4.2) is known exactly and can be manipulated separately. Foss \& Brady (1999) used this decomposition to claim that the long-time self-diffusion coefficient in the $x$-direction can be calculated from only the non-affine part of the motion (denoted $X^{h}$ in (4.2)), specifically from :

$$
D_{x x}=\lim _{t \rightarrow \infty} \frac{1}{2} \frac{\mathrm{d}}{\mathrm{d} t}\left\langle X^{h}(t) X^{h}(t)\right\rangle .
$$


The theoretical analysis of Morris \& Brady (1996) also indicated that such an operation was possible and would result in the correct value of the self-diffusivity for any value of the Péclet number.

A fundamental difference between the shear-induced diffusivity of a non-Brownian suspension and the motion of a Brownian particle is the finite (and large) correlation time for the non-affine displacements. In the case of a Brownian particle, there exists a time interval small enough so that the particle position has not changed significantly, but also large enough so that the random component of the particle's velocity is completely uncorrelated with the random component of the velocity at the previous time interval; this time interval is given by the particle momentum relaxation, $\tau_{B}=m / 6 \pi \eta a$, which is much smaller than the time scale of configurational changes, $a^{2} / D$. Such a time scale does not exist for the case of sheared non-Brownian particles. The hydrodynamic component of the particle velocity, which is what gives rise to diffusive-like motion for sheared suspensions, is only a function of the particle configuration; as a result, in order for this velocity to change significantly (or the velocity autocorrelation function to approach zero), the particle configuration also has to change significantly, and for some cases (e.g. small volume fractions) strains up to 10-20 may be necessary. As was demonstrated in the previous section, this can be a serious limitation for the calculation of $D_{y y}$ and $D_{z z}$ if long enough intervals are not sampled; the consequences are far more apparent, however, for the calculation of $D_{x x}$, since now we also need to consider the coupling between the affine and non-affine motions over the long correlation times.

Starting from the displacement (4.2), the mean-square displacements $\langle x x\rangle$ and $\langle x y\rangle$ can be readily calculated:

$$
\langle x(t) x(t)\rangle=\left\langle X^{h}(t) X^{h}(t)\right\rangle+\left\langle\left(\int_{0}^{t} \dot{\gamma} y(\tau) \mathrm{d} \tau\right)^{2}\right\rangle+2\left\langle X^{h}(t) \int_{0}^{t} \dot{\gamma} y(\tau) \mathrm{d} \tau\right\rangle,
$$

and

$$
\langle x(t) y(t)\rangle=\left\langle X^{h}(t) y(t)\right\rangle+\left\langle y(t) \int_{0}^{t} \dot{\gamma} y(\tau) \mathrm{d} \tau\right\rangle .
$$

Differentiating with respect to time and using (4.5) we have

$$
\frac{\mathrm{d}}{\mathrm{d} t}\langle x(t) x(t)\rangle=\frac{\mathrm{d}}{\mathrm{d} t}\left\langle X^{h}(t) X^{h}(t)\right\rangle+2 \dot{\gamma}\langle x(t) y(t)\rangle+2\left\langle\frac{\mathrm{d} X^{h}(t)}{\mathrm{d} t} \int_{0}^{t} \dot{\gamma} y(\tau) \mathrm{d} \tau\right\rangle,
$$

and

$$
\frac{\mathrm{d}}{\mathrm{d} t}\langle x(t) y(t)\rangle=\frac{\mathrm{d}}{\mathrm{d} t}\left\langle X^{h}(t) y(t)\right\rangle+\dot{\gamma}\langle y(t) y(t)\rangle+\left\langle\frac{\mathrm{d} y(t)}{\mathrm{d} t} \int_{0}^{t} \dot{\gamma} y(\tau) \mathrm{d} \tau\right\rangle .
$$

Although derived for the case of a sheared non-Brownian suspension, the above equations are also valid for the motion of a Brownian particle, where the hydrodynamic displacements $X^{h}$ and $y$ are now replaced by the equivalent random Brownian displacements. The fundamental difference between these two cases lies in the calculation of the last terms in (4.6) and (4.7). For a Brownian particle, the 'diffusive distance' a particle has travelled in the $y$-direction, represented by the integral $\int_{0}^{t} \dot{\gamma} y(\tau) \mathrm{d} \tau$, is uncorrelated with the particle's instantaneous random velocity $\left(\mathrm{d} y / \mathrm{d} t\right.$ or $\left.\mathrm{d} X^{h} / \mathrm{d} t\right)$, since the particle velocities are uncorrelated between time steps $\Delta t$ (the Brownian relaxation time $\tau_{B} \ll \Delta t$, at least within an accuracy $O(\Delta t)$ ). This is not the case for hydrodynamically interacting non-Brownian particles; now a particle's position, or equivalently the distance the particle has travelled in the $y$-direction, is 
strongly correlated with the instantaneous velocity of the particle, since this velocity is purely a result of a particle's position and only changes significantly when the particle configuration changes significantly. It can be argued that this last term will only introduce a contribution of the order of the correlation time, but now the correlation time is large and scales as $\dot{\gamma}^{-1}$, and the last term in (4.6) or (4.7) becomes constant for long times, contributing a linear term to the total mean-square displacement.

The calculation of $\langle x x\rangle$ as given in (4.4) or (4.6) accurately describes the particle displacements and is all the information needed for the motion of the particles in the flow direction. The calculation of a self-diffusion coefficient - corresponding to a 'diffusive'-like term in a master or Fokker-Planck equation - from this meansquare displacement still needs to be addressed, however. It has been suggested (Morris \& Brady 1996; Foss \& Brady 1999) that the self-diffusion coefficient in the longitudinal direction can simply be calculated by only taking into account the nonaffine displacement according to (4.3), or simply the first term on the right-hand side of (4.6). The presence of the last term on the right-hand side of (4.6) or (4.7) makes such a suggestion suspect, and, as will be demonstrated below, the contribution of this term is constant at long times. Note that the second term on the right-hand side of (4.7) is proportional to $\dot{\gamma} D_{y y} t$ at long times and therefore gives a $D_{x y}(=(\mathrm{d} / \mathrm{d} t)\langle x y\rangle / 2)$ growing as $t$ and an $\langle x y\rangle$ growing as $t^{2}$ as in (4.1b). Similarly, the $\langle x y\rangle$-term on the right-hand side of (4.6) gives a $t^{3}$ growth for $\langle x x\rangle$. These are the well-known (Elrick 1962) convectively enhanced mean-square displacements of a (Brownian) particle and cause no conceptual difficulties. Note also that the only non-zero cross-term is $x y$.

To address the issue of the proper definition of the 'diffusivity' in the direction of the shearing motion, we write a master equation for the probability density of finding a particle described by (4.2) (Van Kampen 1992):

$$
\frac{\partial P}{\partial t}=\left(\frac{1}{2} \frac{\mathrm{d}}{\mathrm{d} t}\langle x x\rangle\right) \frac{\partial^{2} P}{\partial x^{2}}+\left(\frac{1}{2} \frac{\mathrm{d}}{\mathrm{d} t}\langle y y\rangle\right) \frac{\partial^{2} P}{\partial y^{2}}+\left(\frac{1}{2} \frac{\mathrm{d}}{\mathrm{d} t}\langle z z\rangle\right) \frac{\partial^{2} P}{\partial z^{2}}+2\left(\frac{1}{2} \frac{\mathrm{d}}{\mathrm{d} t}\langle x y\rangle\right) \frac{\partial^{2} P}{\partial x \partial y},
$$

where $\langle x\rangle=\langle y\rangle=\langle z\rangle=0$, and it has been assumed that the derivatives of the second moments are only functions of time (i.e. they have no spatial dependence). Equation (4.8) simply follows from a second-order expansion of the probability distribution of finding a marked particle and is a very general form valid for a large number of systems. Note that there is no explicit shearing motion, i.e. no advection term in (4.8), because at the macroscopic scale the marked particle has averaged over the microscale shearing flow and wanders 'diffusively'.

The solution of (4.8) can be written as (Van Kampen 1992):

$$
P=\frac{1}{\Delta^{1 / 2}} \exp \left(-\frac{x^{2}}{2 \sigma_{x^{2}}}-\frac{y^{2}}{2 \sigma_{y^{2}}}-\frac{z^{2}}{2 \sigma_{z^{2}}}-\frac{2 x y}{2 \sigma_{x y}}\right),
$$

where

$$
\begin{aligned}
\Delta & =\sigma_{z^{2}}\left(\sigma_{x^{2}} \sigma_{y^{2}}-\sigma_{x y}^{2}\right), \\
\sigma_{x^{2}} & =B_{11}-B_{12}^{2} / B_{22}, \\
\sigma_{y^{2}} & =B_{22}-B_{12}^{2} / B_{11}, \\
\sigma_{z^{2}} & =B_{33}, \\
\sigma_{x y} & =B_{12}-\left(B_{11} B_{22}\right) / B_{12},
\end{aligned}
$$


and $\boldsymbol{B}$ is the matrix of mean-square displacements

$$
\begin{aligned}
& B_{11}=\langle x x\rangle, \\
& B_{22}=\langle y y\rangle, \\
& B_{33}=\langle z z\rangle, \\
& B_{12}=\langle x y\rangle .
\end{aligned}
$$

It is straightforward to verify that (4.9) is a solution of (4.8). This is still a very general result since the functional form for the second moments of (4.11) has not yet been specified. Using the functional forms for $\mathrm{d}\langle x x\rangle / \mathrm{d} t$ and $\mathrm{d}\langle x y\rangle / \mathrm{d} t$ given by (4.6) and (4.7), equation (4.8) can be rewritten as:

$$
\frac{\partial P}{\partial t}=-y \frac{\partial P}{\partial x}+D_{x x}(t) \frac{\partial^{2} P}{\partial x^{2}}+D_{y y}(t) \frac{\partial^{2} P}{\partial y^{2}}+D_{z z}(t) \frac{\partial^{2} P}{\partial z^{2}}+2 D_{x y}(t) \frac{\partial^{2} P}{\partial x \partial y},
$$

where

$$
\begin{aligned}
& D_{x x}(t)=\frac{1}{2} \frac{\mathrm{d}}{\mathrm{d} t}\left\langle X^{h} X^{h}\right\rangle+\left\langle\frac{\mathrm{d} X^{h}}{\mathrm{~d} t} \int_{0}^{t} \dot{\gamma} y(\tau) \mathrm{d} \tau\right\rangle, \\
& D_{y y}(t)=\frac{1}{2} \frac{\mathrm{d}}{\mathrm{d} t}\langle y y\rangle, \\
& D_{z z}(t)=\frac{1}{2} \frac{\mathrm{d}}{\mathrm{d} t}\langle z z\rangle, \\
& D_{x y}(t)=\frac{1}{2} \frac{\mathrm{d}}{\mathrm{d} t}\left\langle X^{h} y\right\rangle+\frac{1}{2}\left\langle\frac{\mathrm{d} y}{\mathrm{~d} t} \int_{0}^{t} \dot{\gamma} y(\tau) \mathrm{d} \tau\right\rangle .
\end{aligned}
$$

Note that the $\langle x(t) y(t)\rangle$ and $\langle y(t) y(t)\rangle$ terms of (4.6) and (4.7) (multiplying the second-order partial derivatives of $P$ ) have been rewritten as the convective flux term $(-y \partial P / \partial x)$ with $P$ given by (4.9). This is a straightforward calculation:

$$
\begin{aligned}
& \frac{1}{2} \frac{\partial^{2} P}{\partial x^{2}} 2\langle x y\rangle+\frac{\partial^{2} P}{\partial x y}\langle y y\rangle \\
&=P\left(\frac{x^{2}}{\sigma_{x^{2}} \sigma_{x^{2}}}+\frac{y^{2}}{\sigma_{x y} \sigma_{x y}}+\frac{2 x y}{\sigma_{x^{2}} \sigma_{x y}}-\frac{1}{\sigma_{x^{2}}}\right)\langle x y\rangle \\
& \quad+P\left(\frac{x y}{\sigma_{x^{2}} \sigma_{y^{2}}}+\frac{x^{2}}{\sigma_{x^{2}} \sigma_{x y}}+\frac{y^{2}}{\sigma_{y^{2}} \sigma_{x y}}+\frac{x y}{\sigma_{x y} \sigma_{x y}}\right)\langle y y\rangle \\
&=P\left(\frac{x^{2} B_{22}^{2} B_{12}}{\left(B_{22} B_{11}-B_{12}^{2}\right)^{2}}+\frac{y^{2} B_{12}^{2} B_{12}}{\left(B_{22} B_{11}-B_{12}^{2}\right)^{2}}-\frac{2 x y B_{22} B_{12}^{2}}{\left(B_{22} B_{11}-B_{12}^{2}\right)^{2}}\right. \\
&-\frac{B_{22} B_{12}}{\left(B_{22} B_{11}-B_{12}^{2}\right)}+\frac{x y B_{22}^{2} B_{11}}{\left(B_{22} B_{11}-B_{12}^{2}\right)^{2}}-\frac{x^{2} B_{22}^{2} B_{12}}{\left(B_{22} B_{11}-B_{12}^{2}\right)^{2}} \\
&\left.-\frac{y^{2} B_{11} B_{12} B_{22}}{\left(B_{22} B_{11}-B_{12}^{2}\right)^{2}}+\frac{x y B_{12}^{2} B_{22}}{\left(B_{22} B_{11}-B_{12}^{2}\right)^{2}}+\frac{B_{22} B_{12}}{\left(B_{22} B_{11}-B_{12}^{2}\right)}\right) \\
&= P \frac{-y^{2} B_{12}+x y B_{22}}{\left(B_{22} B_{11}-B_{12}^{2}\right)},
\end{aligned}
$$

and

$$
-y \frac{\partial P}{\partial x}=P\left(\frac{x y}{\sigma_{x^{2}}}+\frac{y^{2}}{\sigma_{x y}}\right)=P \frac{-y^{2} B_{12}+x y B_{22}}{\left(B_{22} B_{11}-B_{12}^{2}\right)} .
$$


We have denoted the diffusivities as functions of time so that (4.12) and (4.13) are valid at each instant in time, and not only for very long times when the motion becomes truly diffusive. As has been mentioned, at long times, both contributions to $D_{x x}$ and $D_{x y}$ become constant with time - it is those values of the diffusivity that we wish to calculate.

From the master equation (4.8) describing the 'macroscopic' behaviour, we have derived the conventional convection-diffusion equation (4.12) where, by construction, the diffusion coefficients are given by (4.13). It is these diffusion coefficients that we wish to calculate, and it is now apparent that in order to calculate the diffusivity in the $x$-direction we need more information than simply the non-affine displacements. The theoretical analysis of Morris \& Brady (1996) also agrees with the above conclusion; unfortunately, an error in their earlier calculation (which has been corrected) led to the omission of the last terms of (4.13) for $D_{x x}$ and $D_{x y}$.

For simplicity of notation we rewrite (4.13) as:

$$
\begin{aligned}
& D_{x x}(t)=D_{x x}^{\star}(t)+D_{x x}^{c o r r}(t), \\
& D_{x y}(t)=D_{x y}^{\star}(t)+D_{x y}^{c o r r}(t),
\end{aligned}
$$

where

$$
\begin{aligned}
D_{x x}^{\star}(t) & =\frac{1}{2} \frac{\mathrm{d}}{\mathrm{d} t}\left\langle X^{h} X^{h}\right\rangle, \\
D_{x y}^{\star}(t) & =\frac{1}{2} \frac{\mathrm{d}}{\mathrm{d} t}\left\langle X^{h} y\right\rangle,
\end{aligned}
$$

and

$$
\begin{aligned}
& D_{x x}^{\text {corr }}(t)=\left\langle\frac{\mathrm{d} X^{h}}{\mathrm{~d} t} \int_{0}^{t} \dot{\gamma} y(\tau) \mathrm{d} \tau\right\rangle, \\
& D_{x y}^{\text {corr }}(t)=\frac{1}{2}\left\langle\frac{\mathrm{d} y}{\mathrm{~d} t} \int_{0}^{t} \dot{\gamma} y(\tau) \mathrm{d} \tau\right\rangle .
\end{aligned}
$$

We refer to the term omitted by the previous authors as a 'correction' simply for clarity - both terms can be equally important for the shear-induced diffusivity. It can also be seen as a correction compared to the Brownian case in the absence of shear where such a term is absent.

Calculating the self-diffusivities from (4.13) is now straightforward for any simulation. We chose to express these diffusivities as functions of the non-affine displacement because we wanted to stress the importance of the missing term in the calculations of Morris \& Brady (1996) and Foss \& Brady (1999). It is also straightforward to calculate the diffusivities directly from (4.6) and (4.7):

$$
\begin{aligned}
D_{x x}(t) & =\frac{1}{2} \frac{\mathrm{d}}{\mathrm{d} t}\left\langle X^{h} X^{h}\right\rangle+\left\langle\frac{\mathrm{d} X^{h}}{\mathrm{~d} t} \int_{0}^{t} \dot{\gamma} y(\tau) \mathrm{d} \tau\right\rangle \\
& =\frac{1}{2} \frac{\mathrm{d}}{\mathrm{d} t}\langle x(t) x(t)\rangle-\dot{\gamma}\langle x(t) y(t)\rangle,
\end{aligned}
$$

and

$$
\begin{aligned}
D_{x y}(t) & =\frac{1}{2} \frac{\mathrm{d}}{\mathrm{d} t}\left\langle X^{h} y\right\rangle+\frac{1}{2}\left\langle\frac{\mathrm{d} y}{\mathrm{~d} t} \int_{0}^{t} \dot{\gamma} y(\tau) \mathrm{d} \tau\right\rangle \\
& =\frac{1}{2} \frac{\mathrm{d}}{\mathrm{d} t}\langle x(t) y(t)\rangle-\frac{1}{2} \dot{\gamma}\langle y(t) y(t)\rangle .
\end{aligned}
$$


In other words, all the information needed for the calculation of the diffusivities is simply the overall mean-square displacements $\langle x x\rangle,\langle x y\rangle$ and $\langle y y\rangle$. In an experimental set-up, equations (4.13) or (4.17) and (4.18) can be used without further modification. The only requirement is that the particle trajectories must be followed closely enough so that the derivatives and integrals of the mean-square displacements are calculated with sufficient accuracy. Note also, that in general this would be superior to trying to extract from an experiment (or simulation) the linear-in-time terms of the advectively dominated mean-square displacements in (4.1).

Finally, it is important to realize that the time-dependent diffusivities $D_{x x}(t)$ and $D_{x y}(t)$ defined in (4.13) or (4.17) and (4.18) are not necessarily identical at long times to what we would obtain by simply looking at the terms in the mean-square displacements given by (4.1) that grow linearly in time. This can be appreciated by supposing that $D_{y y}(t)$ in $(4.13 b)$ is independent of time and integrating to find

$$
\langle y y\rangle=2 D_{y y} t+a_{y y},
$$

where $a_{y y}$ is a constant of integration. The constant $a_{y y}$ would correspond to the intercept as $t \rightarrow 0$ of the straight line in figure 6 and is a manifestation that the motion is not diffusive at short times. Now, using this in (4.18), assuming $D_{x y}$ is a constant, and integrating gives

$$
\langle x y\rangle=\left(2 D_{x y}+\dot{\gamma} a_{y y}\right) t+D_{y y} \dot{\gamma} t^{2}+b_{x y},
$$

where $b_{x y}$ is another constant and so forth for $\langle x x\rangle$. Thus, a measurement of the linear in time dependence of $\langle x y\rangle$ (assuming this was possible given the domination of the $D_{y y} \dot{\gamma} t^{2}$ term) would yield an apparent $\langle x y\rangle$ diffusivity of

$$
D_{x y}^{\prime}=D_{x y}+\frac{1}{2} \dot{\gamma} a_{y y}
$$

So, is $D_{x y}^{\prime}$ or $D_{x y}$ the correct diffusivity?

From the perspective of the long-time behaviour of the mean-square displacement, we would naturally choose $D_{x y}^{\prime}$. From the perspective of a (possibly time-dependent) diffusivity in the Fokker-Planck equation (4.12), $D_{x y}$ is correct. In other words, since the Fokker-Planck equation involves the time-rate of change of the moments (e.g. $(\mathrm{d}\langle y y\rangle / \mathrm{d} t) / 2$, which are in general functions of time, only approaching a constant in the long-time limit), the mean-square displacements at long time given by the solution of the Fokker-Planck equation are not equal to $D_{x y}$ etc., but to (e.g.) $D_{x y}^{\prime}$, and so the two perspectives are actually in complete agreement. This apparent discrepancy illustrates the importance of distinguishing between a diffusivity, $D_{x y}$, and a meansquare-displacement linear in time, $D_{x y}+\dot{\gamma} a_{y y} / 2$, when advective motion couples to diffusion. We take the definition of the diffusivity (time-dependent or not) as that which appears naturally in the Fokker-Planck equation, with the understanding that we must solve this equation to give the resulting mean-square displacements and they may be different from the underlying diffusivities when advective effects are operative.

Figure 10 shows the $D_{x x}$ self-diffusivity (as calculated by either (4.13) or (4.17) in a simulation where the exact positions are known at each instant in time, the two approaches are identical) as a function of time for a system of $N=1000$ particles and a volume fraction of 0.35 . The curve has been averaged over all particles and over 30 independent configurations. The two separate contributions to the diffusivity, (4.14a), are also shown. Both contributions become constant after the correlation time, 


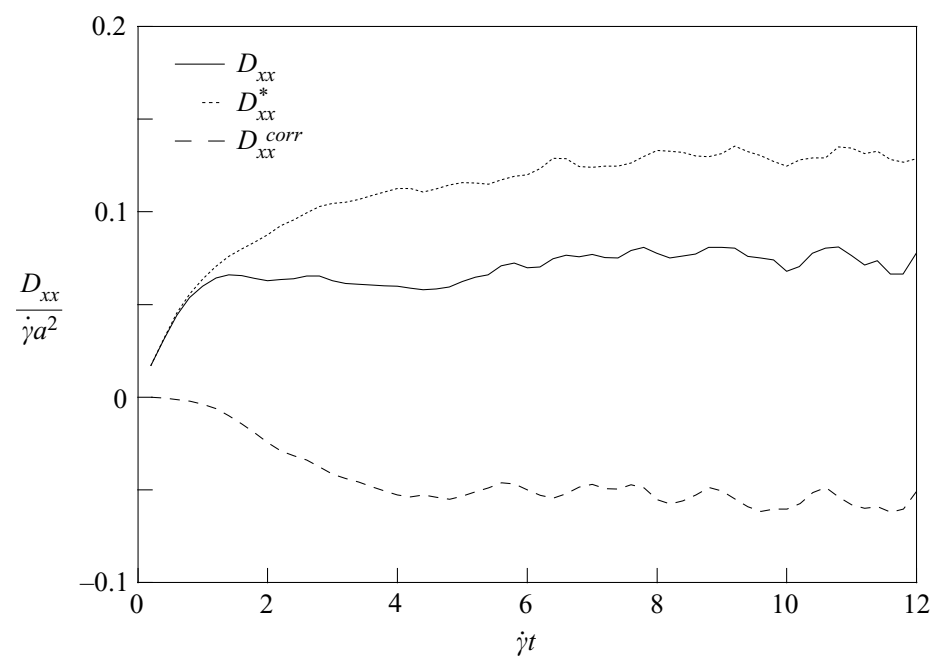

FIGURE 10. The self-diffusivity in the longitudinal direction, $D_{x x}$, is plotted as a function of strain for a system of $N=1000, \phi=0.35$. The values of the two terms contributing to $D_{x x}$ according to $(4.14 a)$ are also given.

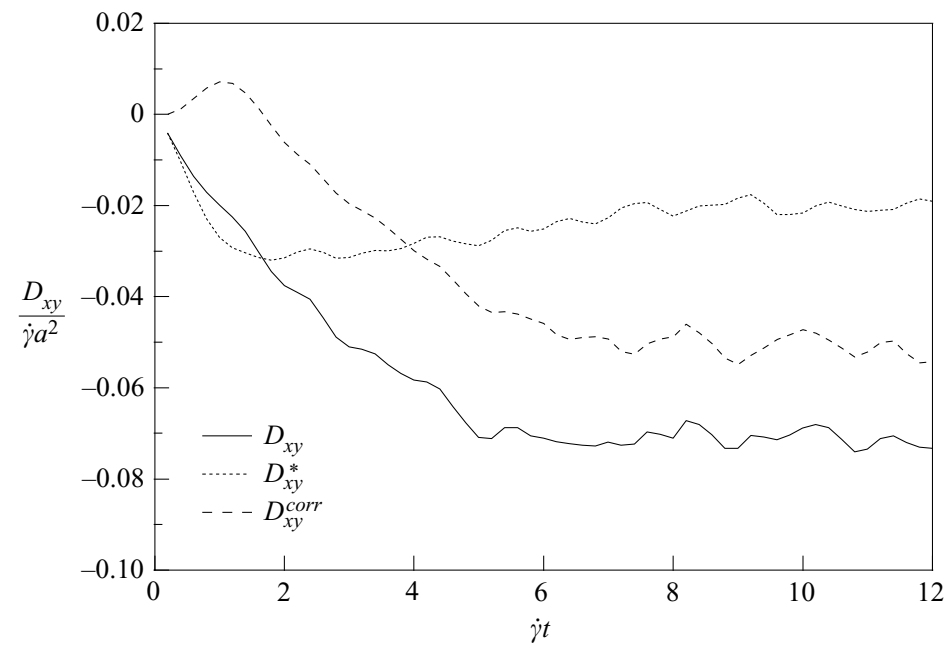

FIgURE 11. The cross-diffusivity, $D_{x y}$, is plotted as a function of strain for a system of $N=1000, \phi=0.35$. The values of the two terms contributing to $D_{x y}$ according to $(4.14 b)$ are also given.

verifying that they both should be included in the calculation of the self-diffusivity. The same behaviour is seen in figure 11, this time for $D_{x y}$. Both corrections to $D_{x x}$ and $D_{x y}$ are negative, resulting in a smaller absolute value of the $D_{x x}$ diffusivity and a larger absolute value of the $D_{x y}$ diffusivity, since the non-affine term, $D_{x y}^{\star}$, is also negative (the physical origin of the negative $D_{x y}$ is discussed in Foss \& Brady 1999). For this relatively high-volume fraction, the effect of the correction to the value of $D_{x x}$ is small. The effect on the value of $D_{x y}$ is rather significant, however, since the non-affine contribution, $D_{x y}^{\star}$, is relatively small. The correction to the diffusivities for a much lower-volume fraction of $\phi=0.15$ is shown in figure 12 . The absolute values of both corrections are now significantly larger, in agreement with the fact that it now 


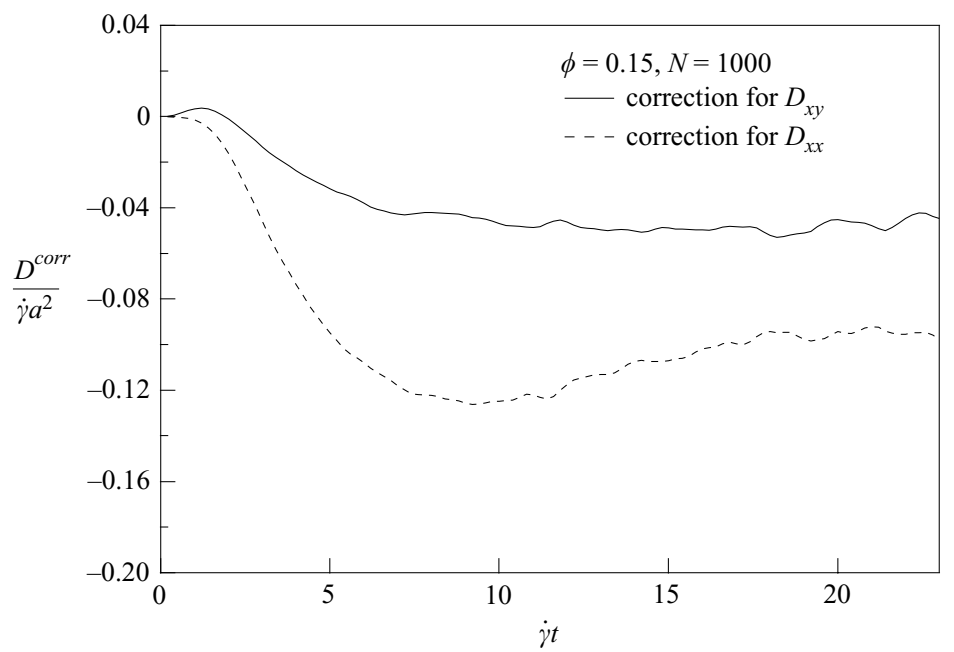

FIGURE 12. The corrective terms, $D_{x x}^{\text {corr }}$ and $D_{x y}^{\text {corr }}$, for a system of $N=1000$ and a relatively small-volume fraction $\phi=0.15$ are plotted as a function of strain.

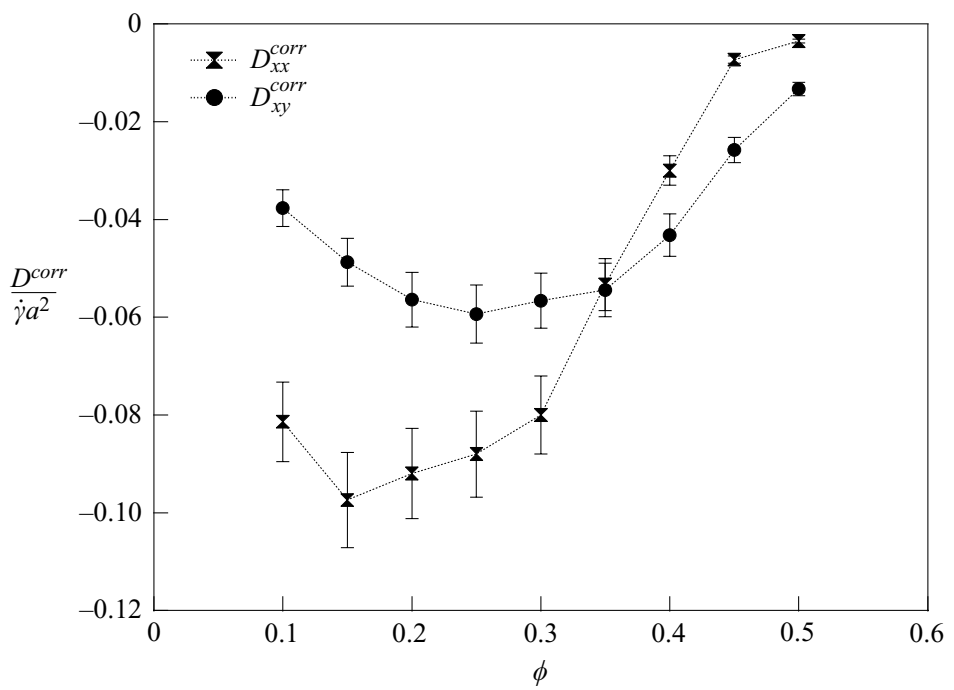

Figure 13. The corrective terms, $D_{x x}^{\text {corr }}$ and $D_{x y}^{\text {corr }}$, for a system of $N=1000$ are plotted as a function of the volume fraction.

takes a much longer time for the particle velocities to become uncorrelated, increasing the time limit for the integrals in $D_{x x}^{\text {corr }}$ and $D_{x y}^{\text {corr }}$; it is also apparent that it takes a longer time for the corrective term to reach a constant value.

For the two-particle problem the non-affine term of the mean-square displacement is well behaved and gives a finite contribution to the diffusivity as $\phi \rightarrow 0$. The contribution $D^{\text {corr }}$, which leads to the singular behaviour observed by Acrivos et al. (1992), can be very important, especially for small-volume fractions, and can dominate the overall behaviour of the mean-square displacement. Figure 13 shows the corrections to the self-diffusivities as a function of volume fraction. Both corrections are increasing (in magnitude) functions of the volume fraction for small volume 
fractions, and decreasing (in magnitude) functions of $\phi$ as the volume fraction is further increased. This behaviour can be explained from the functional form (4.16). The correlation time is a monotonically decreasing function of the volume fraction since it depends directly on the number of collisions between particles. Therefore, the time over which the integrals of (4.16) are calculated decreases as $\phi$ increases. On the other hand, for low-volume fractions, the particle's non-affine $y$ displacement, upon which the corrections to the diffusivity directly depend, are smaller $-D_{y y}$ is small for small-volume fractions. The combination of those two factors results in the observed non-monotonic behaviour for the corrective contribution to the self-diffusivities.

\subsection{The volume fraction dependence}

Before presenting the results for $D_{x x}$, we shall briefly discuss some limitations concerning the calculation of the diffusivity in the flow-direction for small volume fractions. It has been shown by Acrivos et al. (1992) that in the two-particle limit, the behaviour of the mean-square displacement in the $x$-direction is singular and more particles are needed to remove this singularity. Even when the singularity is not present, however, as is the case in our simulations (the singularity is a result of particles coming from infinity interacting with a test particle - in a periodic system, the finite size of the simulation cell introduces a cutoff), the resulting $x$-displacement for a given two-body interaction can be large. For the diffusive regime to be established, each particle must sample a randomly distributed number of different displacements with a zero average. In a dilute periodic system, this implies that each particle, after it interacts with a given second particle and before it again interacts with this particle's image, must sample a sufficient number of interactions with other, third particles. This can give a strict condition on the minimum size of the unit cell, and thus on the size of the system simulated. Although the same condition must be satisfied for concentrated systems, the magnitude of each individual displacement is significantly smaller and their frequency significantly larger at high $\phi$.

To demonstrate the severity of this limitation, consider two particles suspended in a periodic unit cell of finite length $L$, initially at positions with relative displacements $\Delta X_{0}, \Delta Y_{0}$, and for simplicity $\Delta Z_{0}=0$ (as shown in figure 14). If initially in positions with different $y$-coordinates, the two particles will interact and at the end of the interaction will return at the same $y$-positions, but there will be a net displacement in the $x$-direction (Acrivos et al. 1992). After the end of the interaction, the particles will continue moving undisturbed until the 'faster' particle reaches the end of the periodic domain, at which time it will re-enter the cell on the opposite face. For a periodic domain, the maximum distance between two particles has to be less than $L$, so eventually (the time will depend both on $L$ and the difference in the affine undisturbed velocities between the two particles, which is proportional to $\Delta Y_{0}$ ) the two particles will interact again. This second interaction will be identical to the first, since the particles' vertical positions did not change as a result of the first interaction. This behaviour will continue indefinitely and at each 'collision' the displacement of each particle is always exactly the same. It is clear that such a scenario would never evolve into diffusive-like motion, even at infinite time, since only one displacement is sampled for each particle, and of course $\langle\Delta X\rangle \neq 0$.

In a dilute system the same problem is present: a given particle, after interacting with an initial second particle, has a very high probability that it will not collide with any third particle until it exits the periodic cell and interacts with the same particle for the second time. Such a behaviour leads to particle velocities that are strongly correlated for aphysically long times and mean-square displacements that 


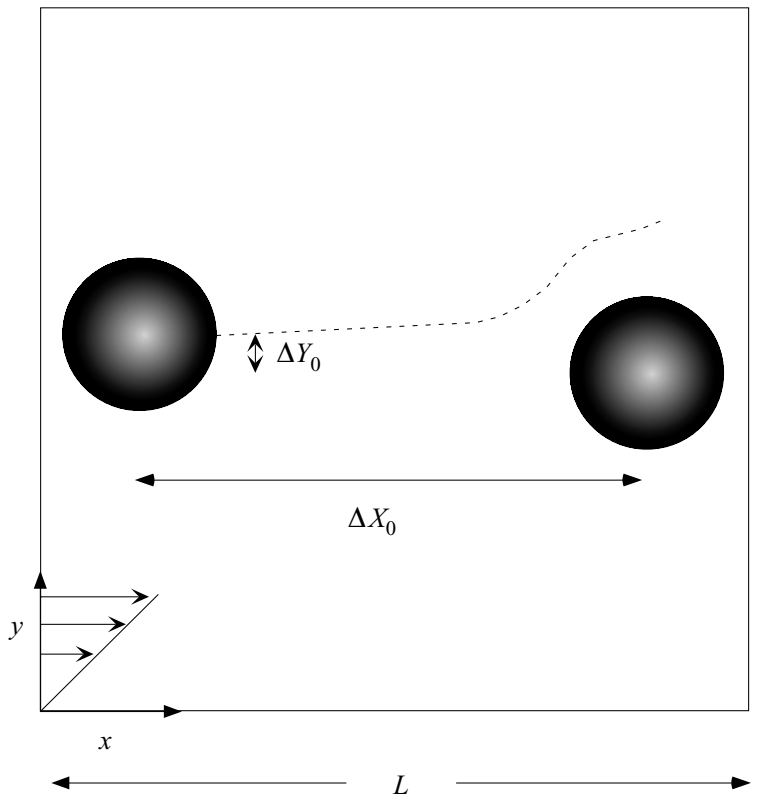

FiguRE 14. Two particles undergoing simple shear flow in a periodic cell of length $L-$ the other two dimensions of the periodic cell are assumed infinite, so that the two particles do not interact with their mirror images in the $y$ - and $z$-directions. The two particles are initially placed at distance $\Delta X_{0}=-10 a, \Delta Y_{0}=0.5 a$ and $\Delta Z_{0}=0$, independently of the value of $L$.

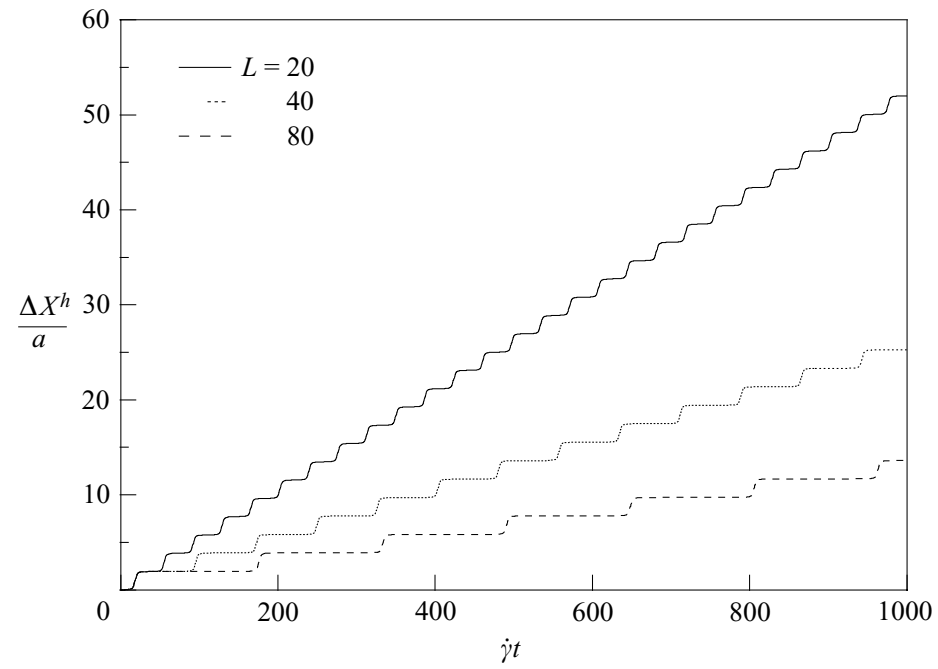

FIGURE 15. The net non-affine displacement $X^{h}$ for one of a pair of particles subject to shear flow is plotted as a function of strain for different values of $L=20 a, 40 a$ and $80 a$.

exhibit quadratic behaviour for very long times. Figure 15 shows the non-affine term $\left(X^{h}\right.$ of (4.2)) of the $x$-displacement for one of the particles of such a pair initially displaced $\Delta X_{0}=-10 a$ and $\Delta Y_{0}=0.5 a$ relative to the reference particle in a unit cell of three different lengths $(L=20 a, 40 a$ and $60 a)$. Each 'step' corresponds to a repeated interaction between the pair of particles resulting in a displacement that appears linear. Increasing the size of the cell simply changes the frequency of each 


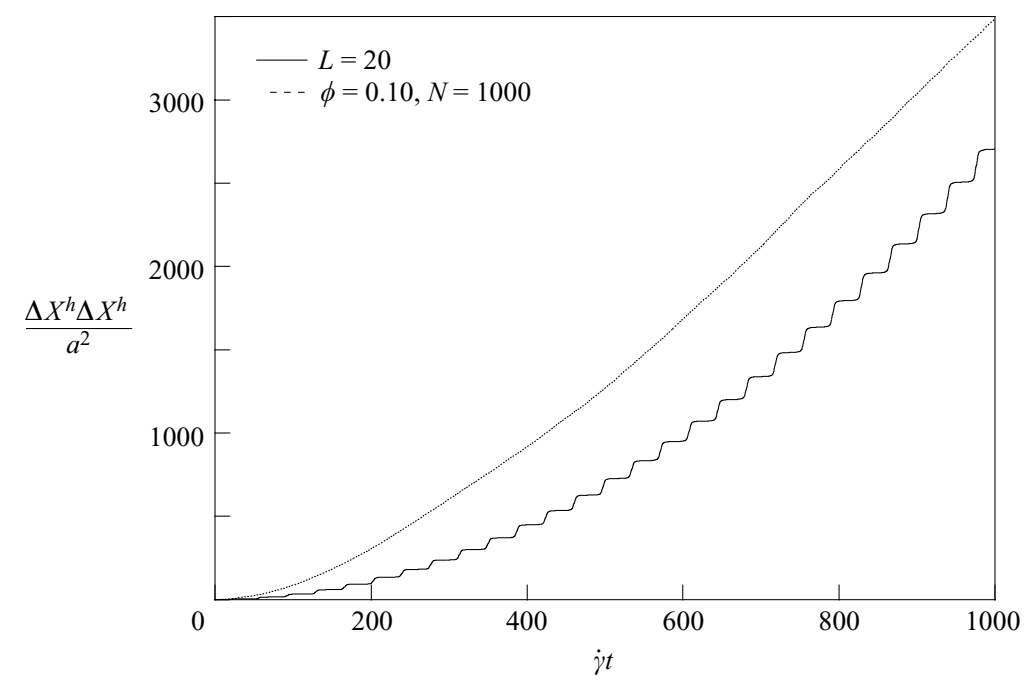

FIGURE 16. The square non-affine displacement $X^{h} X^{h}$ corresponding to the $L=20$ case of the previous figure. The mean-square displacement for a system of 1000 particles at $\phi=0.10$ is also shown.

step, resulting in a linear behaviour with a different coefficient. The mean-square displacement corresponding to the displacement for $L=20 a$ of figure 15 is plotted in figure 16 (we choose to only present the non-affine piece of the displacement, since, as will be shown later, the coupled term has a much more favourable behaviour for non-zero volume fractions). The quadratic behaviour is clear, verifying that such a system of two particles would never reach the diffusive regime. The case studied in figures 15 and 16 corresponds to a given initial lateral displacement between the two particles. Increasing the initial $\Delta Y_{0}$ does not improve the situation because, although each individual step is smaller in magnitude, the frequency is increased; particles that have large $\Delta Y_{0}$ have very different affine velocities and, as a result, approach and interact with each other more frequently. It should be noted that the $\langle y y\rangle$ and $\langle z z\rangle$ mean-square displacements do not suffer from this problem because the net $y$ or $z$ displacements from an encounter are zero, and thus only the 'diffusive' displacements remain. Figure 16 also shows the non-affine term $\left(\left\langle X^{h} X^{h}\right\rangle\right)$ of the meansquare displacement curve for the case of a suspension of $N=1000$ at $\phi=0.10$. The quadratic behaviour is again evident, making it impossible to calculate a diffusion coefficient.

The obvious way to overcome the effect of periodicity is to use a large periodic unit cell. However, this can place a severe requirement on the number of particles needed, especially at low $\phi$. What is necessary is that by the time a particle has crossed the unit cell, its position and velocity should be uncorrelated with its initial position and velocity. Since the particle motion is deterministic, it is the stochastic or shear-induced 'diffusive' motion that results in the loss of correlation. Thus, to lose correlation, a particle must diffuse a distance say $l$ in the $y$-direction before it is convected through the unit cell, i.e. $l^{2} / D_{y y} \ll L / a \dot{\gamma}$, or $L / a \gg \dot{\gamma} l^{2} / D_{y y}$, where $L$ is the length of the unit cell in the $x$-direction. The correlation distance $l$ must be at least of the order of the particle size $a$, and $D_{y y}=\dot{\gamma} a^{2} d_{y y}(\phi)$, so that the simulation cell must satisfy $L / a \gg 1 / d_{y y}(\phi)$. For small $\phi, d_{y y} \sim 10^{-3}$, giving $L / a \sim 10^{3}$ or $N \sim 10^{9}$ for a cubic unit cell. (Attempts to use an elongated non-cubic unit cell experienced 
numerical difficulties associated with shearing a very anisotropic cell.) Even with a fast algorithm, such large-scale simulations are not currently feasible.

In order to obtain meaningful results without using an extremely large system, we followed an alternative approach: we generated a large number of independent configurations of particles and calculated the individual displacements for each individual particle. As has been mentioned, because of the periodicity, the mean displacement for each particle, even after long strains, will not average to zero since particles keep interacting with the same images. In order to obtain the correct behaviour we combine the particles into groups (chosen randomly from different initial configurations) and calculate the mean-square displacements of the resulting groups. If enough particles are in each group, the average displacement for a group fluctuates around zero. (We combine particles from different initial configurations for our averages. Particles from the same configuration could also be used, but there is an upper limit above which the statistics are no longer sampled correctly; e.g. since the suspension is force free, if all the particles are grouped together we obtain not only $\langle x\rangle=0$ but also $\langle x x\rangle=0$.) For the new groups, we calculate the mean-square displacements in all directions and identify a linear regime for the $x$-direction. The times for these simulations are kept relatively short, but long enough to reach, we believe, the diffusive regime. This is done both to reduce the computational cost, since now hundreds of configurations of thousands of particles are used, and to ensure that a linear regime is identified. In order for the diffusive behaviour to be seen, the groups must include enough configurations so that their average displacement is zero. If that is not the case, then as time elapses the systematic error will increase since the individual particle square displacements continue to grow in time. Ideally, we should try to identify a time scale where the diffusion process has started and the particles have not yet left the periodic cell. In order for the diffusion process to be evident, however, many collisions need to occur and therefore the 'grouped' particles need to travel a significant distance, which for the system sizes we are now studying means going through the periodic cell a number of times.

While such a procedure works, it can be subject to large errors, since, on the one hand, grouping of the particles reduces their statistical accuracy (i.e. 10 groups of 1000 particles have the same statistical accuracy of just 10 particles), and, on the other hand, it is not always clear when the motion becomes diffusive, or even whether the grouping, although it improves the behaviour, completely eliminates the problem. Figure 17( $a$ ) shows the derivative of the affine term of the mean-square displacements as a function of time for $\phi=0.15$. A number of independent initial configurations (100) of $N=1000$ particles is used and the results averaged. It is apparent that for a time of order 5-10 strains the behaviour of the mean-square displacement changes dramatically, even without the particle grouping. While the diffusive regime is almost reached, the effect of the periodicity becomes apparent and the diffusivity without grouping becomes linear in time (corresponding to a quadratic behaviour for the mean-square displacement).

Groups of 500 particles are consequently formed (5 particles from each of the independent configurations), and the mean-square displacement of the resulting groups of particles is calculated. Since now the statistics only correspond to the statistics of 200 independent particles, a number (50) of random groupings of the particles are generated and averaged. The effect of the grouping is to transform the linear diffusivity regime into a constant one - as is suggested over the last 10 strains of figure 17(a). Again, there is significant uncertainty as a result of the loss of statistics and the uncertainty associated with the beginning of the linear regime, making it hard 

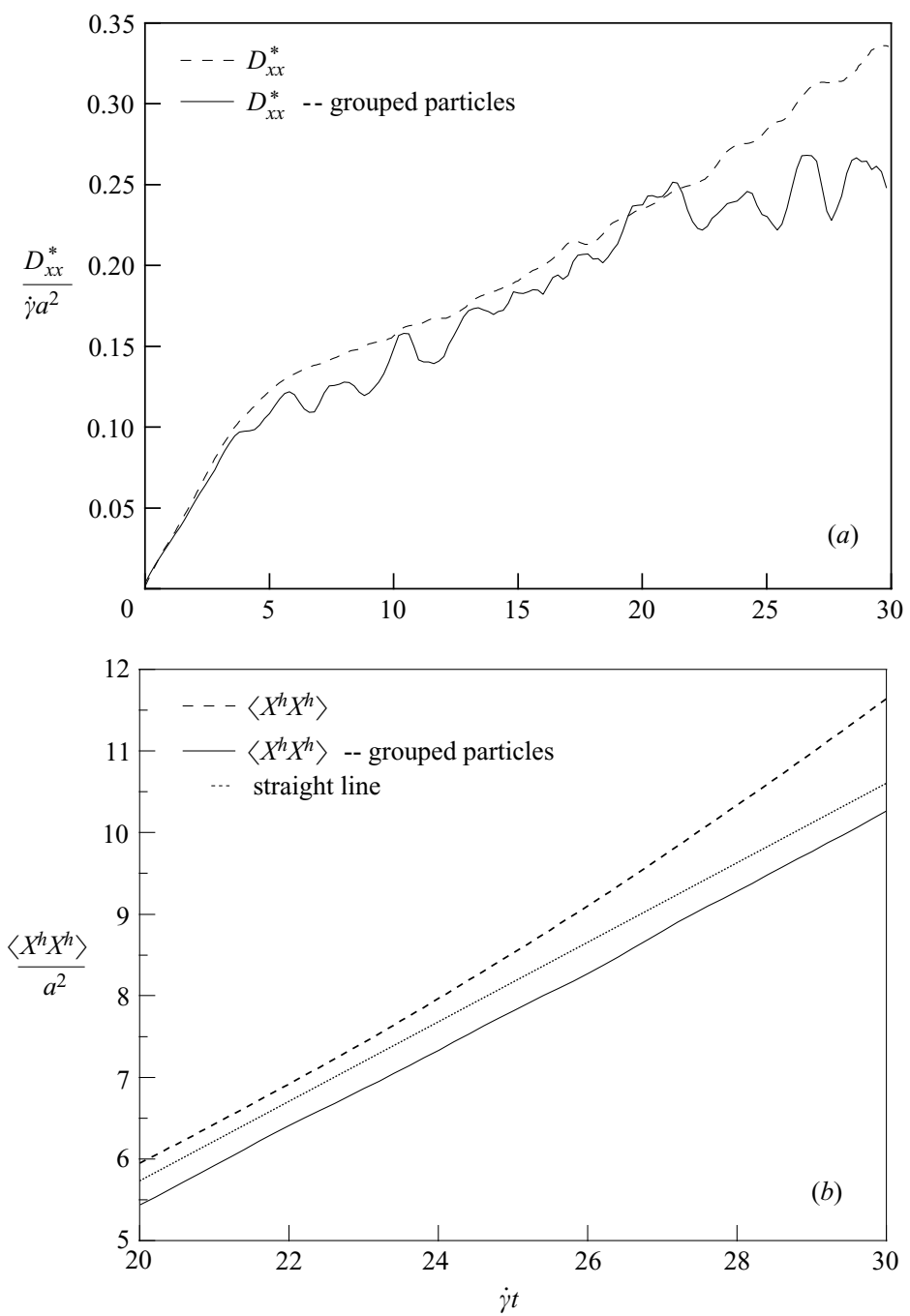

FIGURE 17. The effect of the particle grouping on the calculation of the non-affine diffusivity $D_{x x}^{\star}$. (a) The values of $D_{x x}^{\star}$ are presented as a function of strain for a system of 100 configurations of $N=1000$ at $\phi=0.15$, and compared with the same diffusivity when the particles are first arranged in groups of 500 particles. $(b)$ The corresponding mean-square displacements for these two cases are also presented and compared.

to make solid quantitative estimates. Figure $17(b)$ shows the non-affine mean-square displacement, $\left\langle X^{h} X^{h}\right\rangle$, for the two cases described above for strains between 20 and 30. A linear curve is also shown. It is apparent that the non-grouped curve deviates from the linear behaviour, while the grouped curve seems to follow it for all strains shown. It can also be seen, however, that the difference between the two curves is not dramatic. Despite these limitations, we nevertheless proceed with the calculation of the diffusivities, noting that our results for the smaller-volume fractions may be subject to large errors. We note here that it is $D_{x x}^{\star}$ that has this very strong dependence on periodicity. The other term, $D_{x x}^{\text {corr }}$, plotted in figure 12 for $\phi=0.15$ becomes linear in time. This is due to the coupling with the motion in the $y$-direction, a motion that does not suffer from the same limitations because, even for low-volume fractions, the 


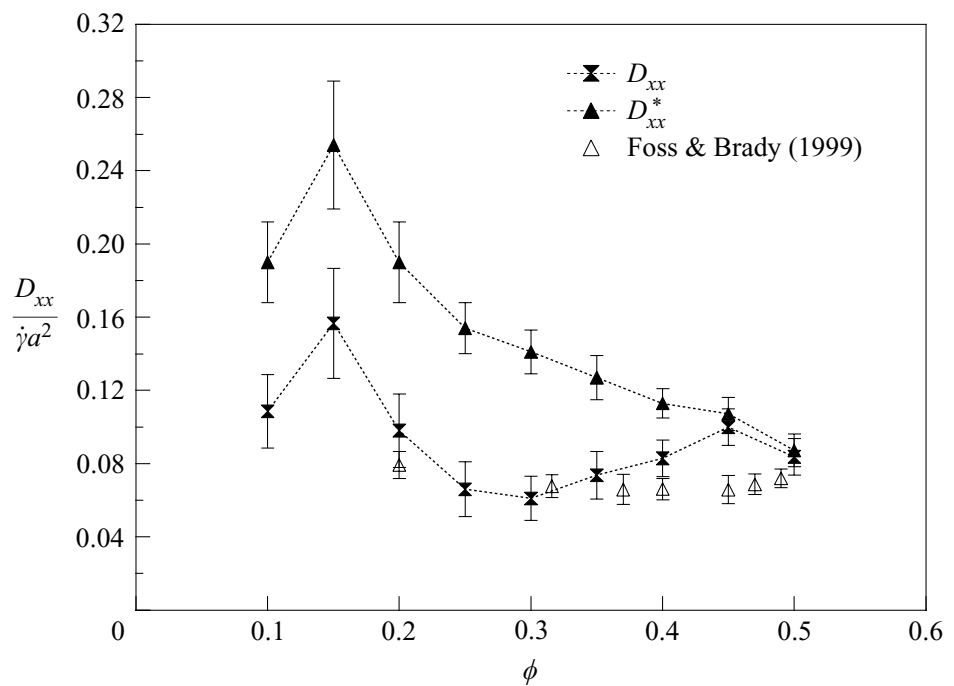

FIGURE 18. The self-diffusion coefficient $D_{x x}$ plotted as a function of the volume fraction. The values of $D_{x x}^{\star}$ are also presented and compared with the simulation results of Foss \& Brady (1999).

$\begin{array}{ccccc}\phi & D_{x x} & D_{x x}^{\star} & D_{x y} & D_{x y}^{\star} \\ 0.10 & 0.1086 \pm 0.020 & 0.190 & -0.0390 \pm 0.004 & -0.0013 \\ 0.15 & 0.1566 \pm 0.030 & 0.254 & -0.0534 \pm 0.005 & -0.0052 \\ 0.20 & 0.0980 \pm 0.020 & 0.190 & -0.0640 \pm 0.007 & -0.0077 \\ 0.25 & 0.0660 \pm 0.015 & 0.159 & -0.0717 \pm 0.009 & -0.0124 \\ 0.30 & 0.0610 \pm 0.012 & 0.141 & -0.0746 \pm 0.010 & -0.0180 \\ 0.35 & 0.0737 \pm 0.013 & 0.127 & -0.0758 \pm 0.009 & -0.0214 \\ 0.40 & 0.0829 \pm 0.010 & 0.113 & -0.0725 \pm 0.007 & -0.0293 \\ 0.45 & 0.0998 \pm 0.010 & 0.107 & -0.0594 \pm 0.007 & -0.0336 \\ 0.50 & 0.0837 \pm 0.010 & 0.087 & -0.0430 \pm 0.006 & -0.0297\end{array}$

TABLE 2. The dependence of the self-diffusion coefficients $D_{x x}$ and $D_{x y}$ on the volume fraction for $N=1000$. The values of $D_{x x}^{\star}$ and $D_{x y}^{\star}$ are also presented.

diffusive motion in the $y$-direction is a result of interaction between at least three particles. The calculation of $\langle x y\rangle$ is also not nearly as problematic as $\langle x x\rangle$, again due to the coupling with the motion in the $y$-direction and the grouping is not necessary.

Figure 18 and table 2 present $D_{x x}$ as a function of the volume fraction, along with the values of $D_{x x}^{\star}$, although as has already been mentioned, $D_{x x}^{\star}$ does not correspond to the self-diffusivity. The diffusivity is expected to be an increasing function of the volume fraction for very dilute systems according to the dilute limit theoretical predictions of Acrivos et al. (1992); this is in agreement with our calculated values between $\phi=0.10$ and $\phi=0.15$. As the volume fraction is increased further, the interactions between particle pairs become more frequent, while at the same time the displacement resulting from a given interaction is expected to decrease in magnitude. The values seem to plateau at higher-volume fractions, at least within the accuracy of our calculations. The volume fraction behaviour of $D_{x y}$, shown in figure 19 and table 2 , is dominated by the behaviour of the corrective term, leading to an initially increasing volume fraction dependence followed by a decreasing one - the value of the 


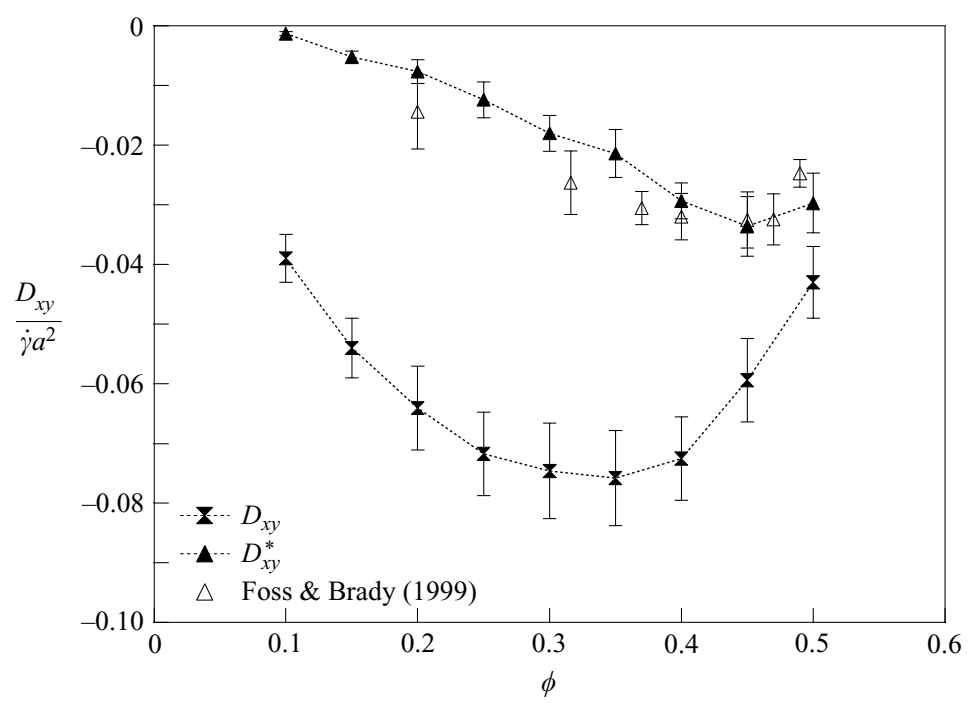

FIGURE 19. The self-diffusion coefficient $D_{x y}$ plotted as a function of the volume fraction. The values of $D_{x y}^{\star}$ are also presented and compared with the simulation results of Foss \& Brady (1999).

non-affine term on the other hand seems to be an increasing function of the volume fraction, reaching a plateau at about the same values where the $D_{y y}$ component also reaches a plateau. Other than $D_{z z}$, the remaining components of the diffusivity tensor have a similar behaviour at high volume fractions - reaching apparent plateaus. This can be expected since there is strong coupling between these three self-diffusivities. The anisotropy between the values of $D_{x x}, D_{y y}$ and $D_{z z}$ decreases with increasing volume fraction - the values of $D_{y y}$ and $D_{z z}$ are very close for $\phi=0.50$, while the value of $D_{x x}$ is less than twice as large. The only other available numerical results for the calculation of these two properties are the results by Foss \& Brady (1999), which are also presented in figures 18 and 19 . Unfortunately, as was already mentioned, the strains used for their study are not sufficiently long and only the $D^{\star}$ term of the diffusivities is calculated in their simulations, making comparisons difficult. To our knowledge, the only experimental results available for $D_{x x}$ and $D_{x y}$ are the preliminary results of Breedveld (2000). The limitation to very small strains before the diffusive behaviour has been established is still present in these results, as was the case for the calculation of $D_{y y}$ and $D_{z z}$. In addition, it is not clear from his analysis whether the correct self-diffusivity, as opposed to $D^{\star}$ or a different combination of the non-affine and affine displacements, is determined. The use of formulae (4.17) and (4.18) should help in the experimental measurement of the diffusivities.

\section{Conclusions}

The calculation of the complete tensor of the shear-induced diffusivities through numerical simulation has been presented. The availability of a faster algorithm allows routine simulations of much larger systems, significantly improving the accuracy of the results and diminishing system size effects. The self-diffusivities in the velocity gradient and vorticity directions, $D_{y y}$ and $D_{z z}$, were calculated as a function of the volume fraction for $\phi=0.10-0.50$ and compared with existing experimental results. While both appear to be increasing functions of the volume fraction for small to intermediate 
$\phi, D_{y y}$ plateaus at high $\phi$, in agreement with a number of previous experiments. The quantitative agreement between our simulation results and experiment was not always satisfactory and we suggest that one of the reasons for this difference is the difficulty in correctly identifying the diffusive regime. The presence of a long correlation time during which the mean-square displacements show first a quadratic and then a transitional behaviour is a main difference between the behaviour of a non-Brownian particle diffusing under the influence of shear and a Brownian particle.

This difference is further manifest in the calculation of the self-diffusivity in the flow direction, $D_{x x}$, and the off-diagonal self-diffusivity, $D_{x y}$. Previous work had suggested that the diffusion in the $x$-direction can be simply calculated by taking into account only the non-affine contributions to the particle velocity (or particle displacement), as would be appropriate for a single Brownian particle. Careful construction of the advection-diffusion or Fokker-Planck equation for the system of sheared nonBrownian particles demonstrated that a coupled term (in addition to the term arising from the non-affine displacements) is present, a term that was erroneously omitted in previous studies. The Fokker-Planck analysis also pointed to the difference between a diffusivity appearing in the advection-diffusion equation and what is obtained from the linear-in-time term of the long-time limit of mean-square displacement. Thus, care is needed when comparing diffusivities obtained from the two approaches. Also, a limitation is present when calculating the longitudinal diffusion coefficient for low volume fractions - the effect of the periodicity is extremely severe leading to an artificial quadratic behaviour in the mean-square displacements. Although an attempt was made to decrease the effect of the periodicity without further increasing the size of the system, the accuracy of the results was not always satisfactory, pointing to the need for either studying larger systems or devising an alternative approach. Very limited experimental data are available for the longitudinal self-diffusivity, and we hope that we have provided a better understanding of how this property is to be defined and can be calculated experimentally via equations (4.17) and (4.18), which would allow meaningful comparisons with experiment in the future.

This work was supported in part by grant NAG8-1661 from NASA and by a Dow graduate fellowship to A. S. Access to an Alpha-based Beowulf cluster at the California Institute of Technology was provided by the Center for Advanced Computing Research. Discussions with G. Subramanian regarding the Fokker-Planck analysis were very helpful.

\section{REFERENCES}

Acrivos, A., Batchelor, G. K., Hinch, E. J., Koch, D. L. \& Mauri, R. 1992 The longitudinal shear-induced diffusion of spheres in a dilute suspension. J. Fluid Mech. 240, 651-657.

Bossis, G. \& Brady, J. F. 1984 Dynamic simulation of sheared suspensions. I. General method. J. Chem. Phys. 80, 5141-5154.

BRADY, J. F. \& Morris, J. F. 1997 Microstructure of strongly sheared suspensions and its impact on rheology and diffusion. J. Fluid Mech. 348, 103-139.

BREedveld, L. V. A. 2000 Shear-induced self-diffusion in concentrated suspensions. PhD thesis, University of Twente, The Netherlands.

Breedveld, V., Van den Ende, D., Bosscher, M., Jongschaap, R. J. J. \& Mellema, J. 2001 Measuring shear-induced self-diffusion in a counter-rotating geometry. Phys. Rev. E 63, $1403-1412$.

Breedveld, V., Van den Ende, D., Tripathi, A. \& Acrivos, A. 1998 The measurement of the shear-induced particle and fluid tracer diffusivities by a novel method. J. Fluid Mech. 375, 297-318. 
DA Cunha, F. R. \& Hinch, E. J. 1996 Shear-induced dispersion in a dilute suspension of rough spheres. J. Fluid Mech. 309, 211-223.

Dratler, D. I. \& SCHOWALter, W. R. 1996 Dynamic simulations of suspensions of non-Brownian hard spheres. J. Fluid Mech. 325, 53-77.

Drazer, G., Koplik, J., Khusid, B. \& Acrivos, A. 2002 Deterministic and stochastic behaviour of non-Brownian spheres in sheared suspensions. J. Fluid Mech. 460, 307-335.

Eckstein, E. C., Bailey, D. G. \& Shapiro, A. H. 1977 Self-diffusion of particles in shear-flow of a suspension. J. Fluid Mech. 79, 191-208.

Elrick, D. E. 1962 Source functions for diffusion in uniform shear flow. Austral. J. Phys. 15, 283-288.

Foss, D. R. \& BRAdy, J. F. 1999 Self-diffusion in sheared suspensions by dynamic simulation. J. Fluid Mech. 401, 243-274.

LeIghton, D. \& ACRIVOS, A. 1987 Measurement of shear-induced migration of particles in concentrated suspensions. J. Fluid Mech. 177, 109-131.

Marchioro, M. \& ACRIVOS, A. 2001 Shear-induced particle diffusivities from numerical simulations. J. Fluid Mech. 443, 101-128.

Melrose, J. R. \& BALL, R. C. 1995 The pathological behaviour of sheared hard-spheres with hydrodynamic interactions. Europhys. Lett. 32, 535-540.

Morris, J. F. \& Brady, J. F. 1996 Self-diffusion in sheared suspensions J. Fluid Mech. 312, 223-252.

Phan, S. E. \& Leighton, D. 1999 Measurement of the shear-induced tracer diffusivity in concentrated suspensions. J. Fluid Mech. (submitted).

Sierou, A. \& Brady, J. F. 2001 Accelerated Stokesian Dynamics simulations. J. Fluid Mech. 448, $115-146$.

Sierou, A. \& Brady, J. F. 2002 Rheology and microstructure in concentrated noncolloidal suspensions. J. Rheol. 46, 1031-1056.

VAN KAMPEN, N. G. 1992 Stochastic processes in physics and chemistry, Rev. and enl. edn. Elsevier.

Wang, Y., Mauri, R. \& Acrivos, A. 1996 Transverse shear-induced liquid and particle tracer diffusivities of a dilute suspension of spheres undergoing a simple shear flow. J. Fluid Mech. 327, 255-272. 